\title{
IRF3 reduces adipose thermogenesis via ISG15- mediated reprogramming of glycolysis
}

\author{
Shuai Yan, ${ }^{1}$ Manju Kumari, ${ }^{1,2}$ Haopeng Xiao, ${ }^{3,4}$ Christopher Jacobs, ${ }^{1,5}$ Shihab Kochumon, ${ }^{6}$ Mark Jedrychowski, ${ }^{3}$ Edward Chouchani, ${ }^{3,4}$ \\ Rasheed Ahmad, ${ }^{6}$ and Evan D. Rosen ${ }^{1,4,5}$ \\ 'Division of Endocrinology, Diabetes, and Metabolism, Beth Israel Deaconess Medical Center, Boston, Massachusetts, USA. ²Department of Biochemistry and Molecular Cell Biology, University Medical \\ Center Hamburg-Eppendorf, Hamburg, Germany. ${ }^{3}$ Department of Cancer Biology, Dana-Farber Cancer Institute, Boston, Massachusetts, USA. ${ }^{4}$ Harvard Medical School, Boston, Massachusetts, USA. \\ ${ }^{5}$ Broad Institute of Harvard and MIT, Cambridge, Massachusetts, USA. ${ }^{9}$ Immunology and Microbiology Department, Dasman Diabetes Institute, Kuwait City, Kuwait.
}

\begin{abstract}
Adipose thermogenesis is repressed in obesity, reducing the homeostatic capacity to compensate for chronic overnutrition. Inflammation inhibits adipose thermogenesis, but little is known about how this occurs. Here we showed that the innate immune transcription factor IRF3 is a strong repressor of thermogenic gene expression and oxygen consumption in adipocytes. IRF3 achieved this by driving expression of the ubiquitin-like modifier ISG15, which became covalently attached to glycolytic enzymes, thus reducing their function and decreasing lactate production. Lactate repletion was able to restore thermogenic gene expression, even when the IRF3/ISC15 axis was activated. Mice lacking ISC15 phenocopied mice lacking IRF3 in adipocytes, as both had elevated energy expenditure and were resistant to diet-induced obesity. These studies provide a deep mechanistic understanding of how the chronic inflammatory milieu of adipose tissue in obesity prevents thermogenic compensation for overnutrition.
\end{abstract}

\section{Introduction}

Body weight is under homeostatic control, in that both gain and loss of body weight initiate physiological events that tend to restore energy balance to normal (1). Thus, short-term weight loss induced by food restriction is countered by reductions in energy expenditure $(2,3)$, and conversely, short-term "experimental obesity" induces reduced appetite and increased energy expenditure $(4,5)$. Despite this, we are in the midst of an obesity epidemic associated with the recent availability of cheap, energy-dense foods. Why don't our bodies compensate for chronic overnutrition with a concomitant elevation in energy expenditure? It is notable that obese humans and animals show reduced adipose thermogenesis $(6,7)$, rather than the enhanced activity one might expect. Identifying the causes of this paradoxical response may provide new clues to the pathophysiology and therapy of obesity.

White adipose tissue undergoes numerous changes in the setting of overnutrition, including the development of a low-grade inflammatory state associated with the acquisition of activated immune cells and the onset of insulin resistance. The mechanistic connections between these phenomena have been a focus of adipose tissue biologists for decades (8). Thermogenic brown and beige adipocytes are also affected by the altered immune state during overnutrition, with most studies showing that chronic inflammation diminishes heat production. This effect is linked to the production of macrophagederived cytokines like TNF- $\alpha$ and IL- $1 \beta$, which impair beige adipogenesis, promote brown adipocyte apoptosis, and reduce the expression

Conflict of interest: The authors have declared that no conflict of interest exists. Copyright: () 2021, American Society for Clinical Investigation.

Submitted: October 5, 2020; Accepted: February 10, 2021; Published: April 1, 2021.

Reference information: J Clin Invest. 2021;131(7):e144888.

https://doi.org/10.1172/JCI144888. of thermogenic genes in both brown and beige adipocytes (9-13). In addition to receptors for classic cytokines, thermogenic adipocytes also express Toll-like receptors (TLRs) and other pattern recognition receptors, which decrease the expression of the uncoupling protein 1 (Ucp1) gene and heat production when activated $(14,15)$.

The transcriptional mechanisms by which cytokines and TLR ligands exert their effects on thermogenesis are not well understood. There has been an assumption that nuclear factor- $\kappa \mathrm{B}(\mathrm{NF}-\kappa \mathrm{B})$, known to mediate many transcriptional events in response to activation of cytokine and TLR pathways, must be involved in the repression of thermogenesis. Interestingly, activation of NF- $\mathrm{BB}$ by overexpression of the active p65 subunit in adipocytes increased energy expenditure and insulin sensitivity, despite a proinflammatory gene expression pattern (16). Similarly, mice lacking the regulatory p50 subunit have increased NF- $\kappa \mathrm{B}$ activity, and display resistance to diet-induced obesity and increased energy expenditure $(17,18)$. These studies suggest that NF- $\mathrm{kB}$-independent transcriptional pathways may mediate the effect of inflammation on thermogenic gene expression.

Another class of transcription factors with major functions in both innate and adaptive immunity are the interferon regulatory factors (IRFs), comprising 9 members (IRF1-IRF9). IRFs have been implicated in almost every aspect of immune function, such as macrophage development, lymphopoiesis, and antiviral defense (19). We have previously demonstrated that several IRFs regulate adipogenesis (20), and we have also elucidated a role for IRF4 as a prolipolytic and prothermogenic partner of PGC- $1 \alpha$ in brown and beige adipocytes $(21,22)$. More recently, we determined that the expression of IRF3, a key regulator of type I IFN gene expression induced in response to pathogenic infection, is elevated in the adipose tissue of obese rodents and humans (23). Furthermore, global $\mathrm{Irf3}^{-/-}$mice are protected from high-fat diet-induced (HFDinduced) obesity and insulin resistance, and exhibit increased 
energy expenditure attributable to enhanced browning of white adipose tissue (23). It is unclear, however, whether this effect of IRF3 is due to its role within adipocytes. More importantly, we still do not know which target genes of IRF3 reduce thermogenesis, or the mechanism by which this occurs.

Here we find that adipocyte-specific Irf3 deficiency attenuates HFD-induced obesity and glucose intolerance, as a result of dramatically increased beige adipocyte thermogenesis. Conversely, adipocyte-specific overexpression of Irf3 suppresses thermogenesis and promotes obesity and insulin resistance. Furthermore, we identify the small ubiquitin-like protein ISG15 as a critical downstream target of IRF3. ISG15 mediates the effect of IRF3 on thermogenesis by reprogramming adipocyte metabolism, through covalent inhibition of glycolytic enzymes and reduction of lactate. Remarkably, loss of Isg15 in vivo increases adipose thermogenesis and protects mice from HFD-induced obesity and glucose intolerance. These findings uncover a new role for IRF3 and ISG15 in the regulation of glucose metabolism and adaptive thermogenesis.

\section{Results}

Adipocyte-specific knockout of Irf 3 increases browning of white adipose tissue. To investigate the role of IRF3 in adipocytes in vivo, we generated mice bearing a floxed $\operatorname{Irf} 3$ allele $\left(\operatorname{Irf} 3^{f l o x}\right)$ (Figure 1A). These mice were crossed to Adipoq-cre ${ }^{+}$animals (22) to create fat-specific IRF3-knockout mice (FI3KO). Irf3-floxed mice (Irf $3^{f / f l}$ Adipoq-cre-) were used as controls, and are hereafter designated as wild type (WT). As expected, $\operatorname{Irf} 3$ mRNA expression and protein levels were significantly lower in primary adipocytes isolated from inguinal and epididymal white adipose tissue (WAT) of FI3KO mice versus WT mice (Figure 1, B and C, and Supplemental Figure 1A; supplemental material available online with this article; https:/doi.org/10.1172/ JCI144888DS1). This pattern was seen only in adipocytes, as $\operatorname{Irf3}$ expression was not affected in the stromal-vascular fraction (SVF) of inguinal WAT (iWAT) and epididymal WAT (eWAT) (Supplemental Figure 1, B and C) or in other tissues such as liver and muscle (Supplemental Figure 1D). Importantly, the global Irf3-knockout mouse also has an inadvertent deletion of a nearby gene, Bcl2like-12 ( $B c l 2 l 12)$, encoding a protein that functions as an antiapoptotic factor (24-26). Our conditional allele does not share this problem, as Bcl2l12 mRNA levels in primary adipocytes of FI3KO mice were comparable to those of WT controls (Supplemental Figure 1E).

We placed 8-week-old male FI3KO and control mice at $4^{\circ} \mathrm{C}$ for 7 days and then assessed the expression of cold-induced thermogenic genes in iWAT and brown adipose tissue (BAT). Quantitative PCR (qPCR) analysis confirmed that many thermogenic markers were significantly increased in iWAT (Figure 1D), but not in BAT (Supplemental Figure 1F), from chow-fed FI3KO mice. UCP1 protein levels were also markedly higher in iWAT, but not BAT, as a result of adipocyte Irf3 deficiency (Figure 1E and Supplemental Figure 1G). Consistent with this, histology revealed increased numbers of multilocular $\mathrm{UCP}^{+}$adipocytes in the iWAT of FI3KO mice (Figure 1F). These results demonstrate that adipocyte IRF3 represses beiging of iWAT.

Adipocyte-specific knockout of Irf3 attenuates HFD-induced obesity in mice and increases insulin sensitivity. To assess the metabolic consequences of IRF3 knockout in adipocytes, we exposed 8-week-old male FI3KO and control mice to a high-fat diet (HFD; for 16 weeks. Notably, FI3KO mice displayed reduced body weight that became apparent after 12 weeks of high-fat feeding (Figure 2A). Echo-MRI analysis of FI3KO mice after 16 weeks of HFD revealed decreased fat mass with no difference in lean mass (Figure 2B). Specifically, the weight of iWAT and liver was significantly reduced in FI3KO mice on HFD (Figure 2C). Histological analysis also revealed that iWAT from FI3KO contained smaller adipocytes (Figure 2D and Supplemental Figure 2A). Furthermore, FI3KO mice had diminished hepatic steatosis, as shown histologically and by measurement of liver triglyceride content (Figure 2, E and F).

To establish how adipocyte Irf3 deficiency affects whole-body energy balance, we performed indirect calorimetry on WT and FI3KO mice fed HFD for 4 weeks only, to minimize confounding by altered body weight. Compared with control mice, FI3KO mice exhibited significantly elevated oxygen consumption and energy expenditure at both room temperature and $4^{\circ} \mathrm{C}$ (Figure $2 \mathrm{G}$ and Supplemental Figure 2B). These results suggest that adipocyte $\operatorname{Irf} 3$ affects beige adipocyte function in vivo and that beige adipocytemediated adaptive thermogenesis plays an important role in the protection against diet-induced obesity observed in our mouse model. To confirm this, we housed mice at $30^{\circ} \mathrm{C}$, at which there is no requirement for adaptive thermogenesis to maintain body temperature (27). Under thermoneutral conditions, there were no differences in oxygen consumption and energy expenditure between WT and FI3KO mice (Figure 2G and Supplemental Figure 2B).

FI3KO mice showed improved glucose and insulin tolerance at 16 weeks of HFD feeding (Supplemental Figure 2, C and D). In order to confirm that the difference in glucose and insulin tolerance seen at 16 weeks of high-fat feeding did not reflect altered adiposity between WT and FI3KO mice, we repeated these experiments on a new cohort of mice after only 8-9 weeks of high-fat feeding, at which point adiposity was equivalent between WT and FI3KO mice (Figure 2A). Again, FI3KO mice exhibited significantly greater insulin and glucose tolerance with markedly lower fed and fasting insulin levels (Figure 2, H and I, and Supplemental Figure 2E). Together these results suggest that adipocyte IRF3 deficiency protects against diet-induced insulin resistance independent of body weight.

Adipocyte-specific overexpression of Irf3 suppresses browning of white adipose tissue. Mutation of Ser396 and Ser398 of human IRF3 to Asp results in a constitutively active allele without the need for an external stimulus like lipopolysaccharide (LPS) or poly I:C (28). We have shown that the analogous double-mutant murine Irf3 allele (S388D/S390D; hereafter designated as IRF32D) is constitutively active in vitro (23). To further investigate the role of adipocyte IRF3 in adaptive thermogenesis, we developed a line of knock-in mice in which the IRF3-2D allele was inserted downstream of the ROSA26 promoter locus behind a loxP-STOPloxP cassette (Supplemental Figure 3A). Crossing to Adipoq-Cre mice enables targeted expression of IRF3-2D in adipocytes (22). Mice that carry both Adipoq-Cre and R26-LSL-FLAG-IRF3-2D transgenes (hereafter designated as fat IRF3 overexpressors, or FI3OE) exhibited moderate (about 3- to 6-fold) overexpression of Irf 3 in adipose tissue (Figure 3, A and B, and Supplemental Figure $3 \mathrm{~B})$, which is similar to the amount of IRF3 induction seen in WAT following exposure to HFD in WT mice (23). FI3OE mice showed lower expression of UCP1 and other thermogenic markers after 7 days of cold challenge in iWAT, but not in BAT (Figure 3, C and D, and Supplemental Figure 3, C and D). 
A IRF3 WT allele

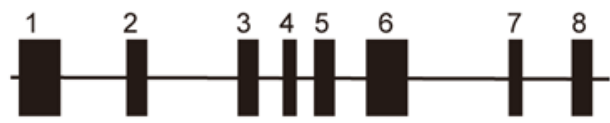

IRF3 floxed allele

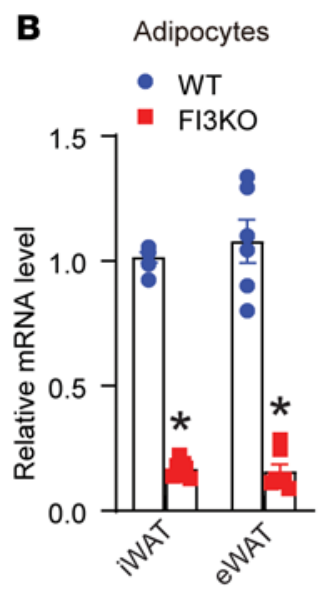

D

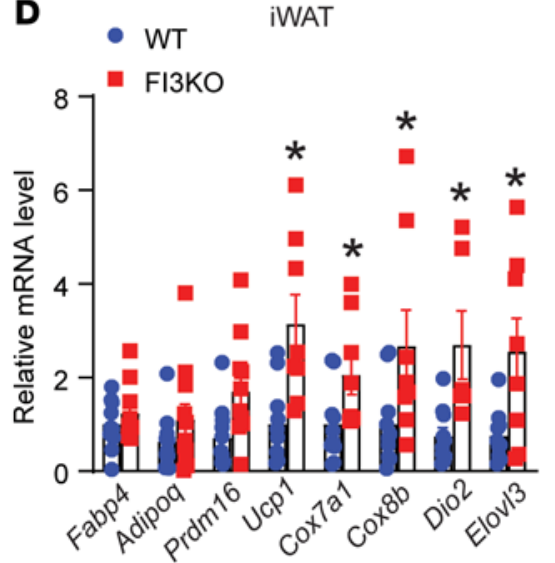

$\mathbf{E}$
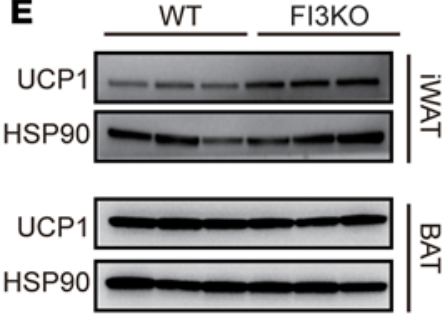

C Adipocytes

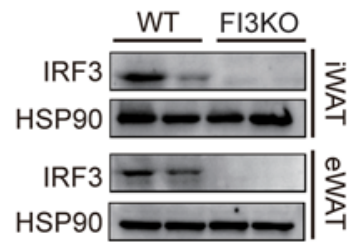

$\mathbf{F}$
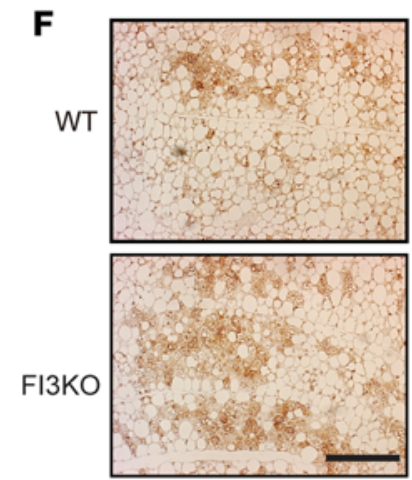

Figure 1. Adipocyte-specific IRF3 deficiency increases thermogenic gene expression. (A) Schematic diagram showing generation of FI3KO mice. (B) Irf3 mRNA expression in isolated primary adipocytes from inguinal white adipose tissue (iWAT) and epididymal white adipose tissue (eWAT) of 8-week-old WT and FI3KO mice $(n=6)$. (C) Western blot of IRF3 protein in SVF from iWAT and eWAT of 8-week-old WT and FI3KO male mice ( $n=3$ ). (D) Thermogenic gene expression in brown adipose tissue (BAT) of chow-fed WT and FI3KO mice after 7 days of cold challenge $(n=8-10)$. (E) Western blot of UCP1 in iWAT and BAT of mice as described in $\mathbf{D}(n=3)$. (F) UCP1 staining of iWAT of WT and FI3KO mice as described in $\mathbf{D}$. Scale bar: $100 \mu \mathrm{m}$. Statistical comparisons were made using 2-tailed Student's $t$ test $(\mathbf{B}$ and $\mathbf{D})$. All data are mean $\pm \mathrm{SEM}$. ${ }^{*} P<0.05$.

Consistent with these findings, histological analysis revealed diminished multilocular UCP1 ${ }^{+}$cells in iWAT of FI3OE mice (Figure 3E). Similarly, treatment with the $\beta_{3}$-agonist CL316,243 markedly increased the expression of thermogenic markers and accumulation of beige adipocytes in iWAT of control mice, but this activity was suppressed in FI3OE mice (Supplemental Figure 3, $\mathrm{E}-\mathrm{G})$. Thus, adipocyte-specific activation of Irf3 suppresses adaptive thermogenesis in iWAT.

Adipocyte-specific overexpression of Irf3 increases diet-induced obesity and promotes insulin resistance. To determine the effect of adipocyte-specific overexpression of Irf3 on glucose homeostasis, we challenged WT and FI3OE mice with HFD for 16 weeks. Notably, weight gain (Figure 4A) and adiposity (Figure 4B and Supplemental Figure 4A) were more prominent in FI3KO mice than in WT mice, which was mainly attributable to the expansion of iWAT (Figure 4C). Furthermore, lipid accumulation in the liver was significantly higher in FI3OE than in WT mice, as shown histologically and by measurement of liver triglyceride content (Figure 4, D and E). A higher density of crown-like structures was noted in the eWAT of FI3OE mice after HFD feeding (Figure 4F). Consistent with this, robust increases in the expression of signature M1 marker genes
(Tnf, Il6, and Nos2) and significant decreases in the expression of M2 markers (Ym1, Arg1, and Il1O) were seen in both eWAT and iWAT SVF of FI3KO mice (Supplemental Figure 4, B and C). These data suggest that adipocyte Irf3 overexpression increases diet-induced obesity and chronic inflammation in eWAT and iWAT.

There was no difference in food intake or glucose and insulin tolerance between WT and FI3OE mice maintained on a chow diet (Supplemental Figure 4, D-F). However, after 16 weeks of HFD, FI3OE mice exhibited markedly impaired insulin and glucose tolerance (Supplemental Figure 4, G and H). Again, we repeated these experiments on a new cohort after 6 weeks of HFD feeding, before divergence of body weight or adiposity; FI3OE mice still showed significantly impaired insulin and glucose tolerance (Figure 4, G and H) with markedly higher fed and fasting plasma insulin levels (Supplemental Figure 4I). Taken together, these results demonstrate that adipocyte-specific Irf 3 overexpression increases HFD-induced obesity and impairs insulin sensitivity. Furthermore, at least some part of the insulin resistance associated with IRF3-2D overexpression in fat is independent of the development of excess body weight.

ISG15 is responsible for IRF3-mediated impaired thermogenesis. IRF3 is a transcription factor, and to identify downstream target 

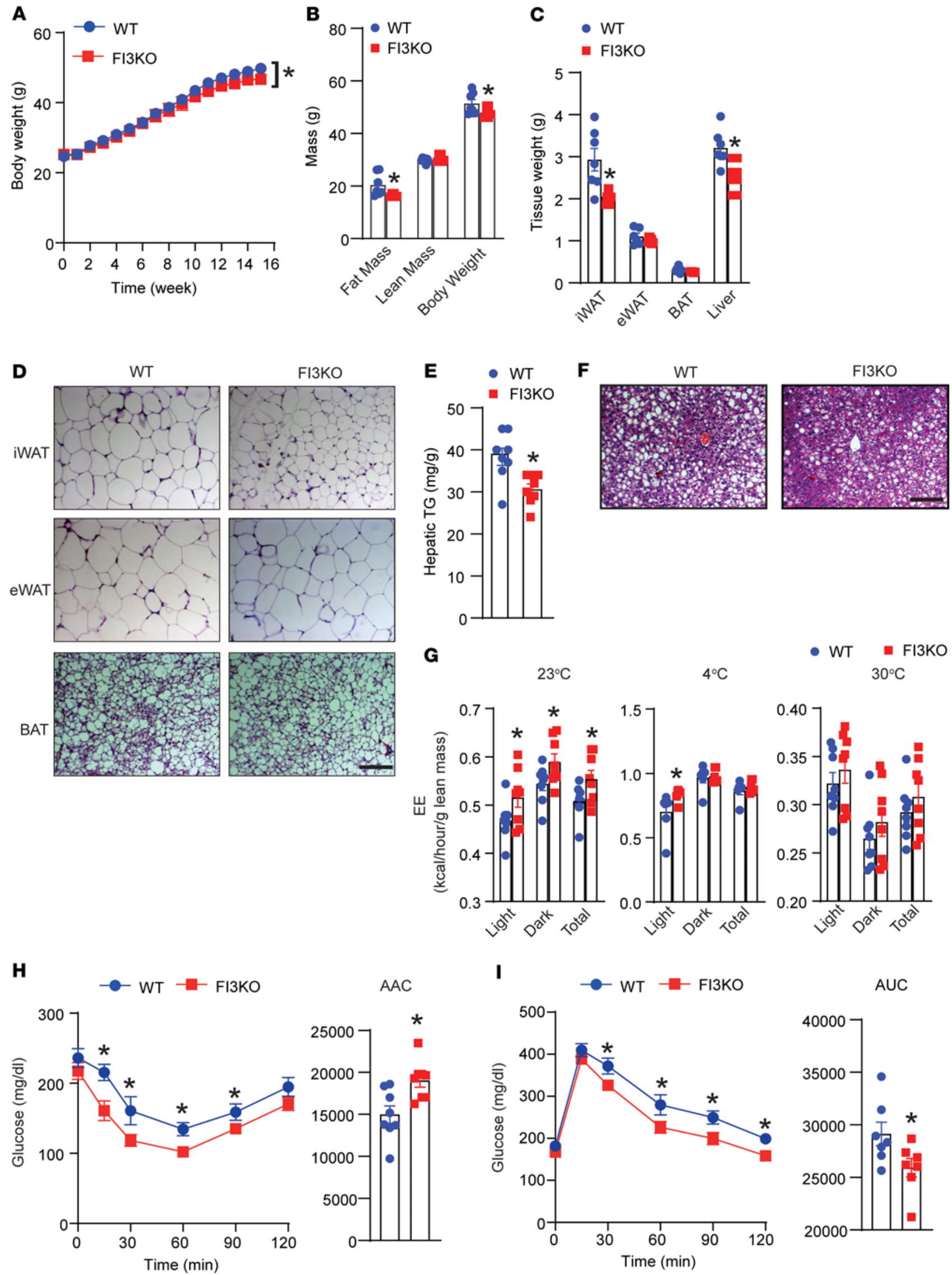

AUC

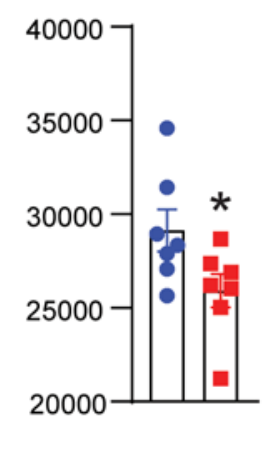


Figure 2. Adipocyte-specific IRF3 deficiency attenuates HFD-induced obesity. (A) Body weight of male WT and FI3KO mice during high-fat feeding ( $n=8-10)$. (B) Body composition of male WT and FI3KO mice after 16 weeks of high-fat feeding $(n=8-10)$. (C) Adipose depot and liver weight of male WT and FI3KO mice after 16 weeks of high-fat feeding $(n=8-10)$. (D) H\&E staining of adipose tissues of WT and FI3KO mice after 16 weeks of high-fat feeding. Scale bar: $200 \mu \mathrm{m}$. (E and F) Hepatic triglyceride (TC) (E) and H\&E staining of liver (F) of WT and FI3KO mice after 16 weeks of HFD feeding ( $n=8-10)$. Scale bar: $200 \mu \mathrm{m}$. (G) Energy expenditure (EE) of male WT and FI3KO mice after 4 weeks of HFD feeding $(n=8-10)$. (H) Insulin tolerance test (ITT) performed in male WT and FI3KO mice after 8 weeks on HFD. Right panel: Area above the curve (AAC) of ITT ( $n=8-10)$. (I) Glucose tolerance test (GTT) performed in male WT and FI3KO mice after 8 weeks on HFD. Right panel: Area under the curve (AUC) of GTT $(n=8-10)$. Statistical comparisons were made using 2-way ANOVA (A, $\mathbf{H}$, and I) or 2-tailed Student's $t$ test (B, C, E, and $\mathbf{G})$. Data are presented as mean \pm SEM. ${ }^{*} P<0.05$.

genes that mediate its actions on thermogenesis, we defined the transcriptional profiles of primary iWAT adipocytes from chowfed WT and FI3OE mice by RNA sequencing (RNA-Seq). Aside from Irf3 itself, two of the most upregulated genes in FI3OE adipose tissue were Isg15 and Herc6 (Figure 5A and Supplemental Table 1). This was confirmed using qPCR of primary adipocytes from FI3OE mice (Figure 5B). Furthermore, Isg15 and Herc6 were downregulated in adipocytes from FI3KO mice (Figure 5C). Ubiquitin-like protein ISG15 is an interferon-induced protein that has been implicated as a central player in the antiviral response (29). Conjugation of ISG15 to target proteins, referred to as ISGylation, utilizes a 3-step enzymatic cascade similar to that of ubiquitination, with the final step catalyzed in mice by the HECT domaincontaining E3 ligase HERC6 (30). To demonstrate that adipocytes support protein ISGylation, we treated primary adipocytes with LPS, and noted marked induction of both free ISG15 and ISGylated protein conjugates (Supplemental Figure 5A). Isg15 and Herc6 were markedly suppressed in primary adipocytes of iWAT from WT mice by cold exposure; FI3KO mice showed a further reduction in levels of these mRNAs at both room temperature and $4^{\circ} \mathrm{C}$ (Supplemental Figure 5B). Based on these observations, we hypothesized that aberrant or excessive ISGylation might account for Irf3-mediated suppression of adaptive thermogenesis.

First, we aimed to determine the requirement for ISG15 in Irf3-mediated suppression of adaptive thermogenesis in vitro. Primary beige adipocytes were generated by differentiation of primary SVF cells from the iWAT of WT and $I s g 15^{-/-}$mice. Importantly, ablation of Isg15 did not affect beige adipogenesis in vitro, as evidenced by qPCR analysis of adipogenic markers and Oil Red $\mathrm{O}$ staining (Supplemental Figure 5, C and D). However, deficiency of Isg15 significantly increased UCP1 protein levels (Figure 5D and Supplemental Figure $5 \mathrm{E}$ ) in beige adipocytes. Isg15 ${ }^{-/}$beige adipocytes also displayed a higher basal oxygen consumption rate (OCR) than did WT beige adipocytes, as well as elevated coupled, uncoupled, and maximal respiration (Figure 5E). Conversely, adenovirus-mediated overexpression of ISG15 markedly suppressed cellular OCR in WT beige adipocytes (Figure 5F). These findings demonstrate that ISG15 can suppress mitochondrial respiration in a cell-autonomous manner.

We next tested whether ISG15 is required for IRF3-mediated suppression of thermogenic gene expression. $\mathrm{Irf3}^{-/-}$primary beige adipocytes have elevated UCP1 protein levels, which were suppressed by overexpression of ISG15 mediated by adenoviral delivery (Figure $5 \mathrm{G}$ and Supplemental Figure 5, F and G). Consistent with these results, $\operatorname{Irf} 3^{-/}$primary beige adipocytes demonstrated elevated OCR, which was reduced by overexpression of ISG15 (Figure $5 \mathrm{H}$ ). We also assessed the converse situation: reduced UCP1 protein and OCR were detected in primary beige adipocytes differentiated from the iWAT SVF of FI3OE mice (Figure 5, I and J, and Supplemental Figure 5, $\mathrm{H}$ and I). These effects were reversed by ectopic expression of USP18, the dominant mammalian de-ISGylase (Figure 5, I and J, and Supplemental Figure 5, H and I) (31).

To determine whether the IRF3/ISG15 pathway affects thermogenesis in human adipocytes, we used a human brown fat cell line (32). LPS treatment markedly increased expression of ISG15 and HERC5 (the human E3 ISG15 ligase corresponding to Herc6 in mice). LPS also increased both free ISG15 and ISGylated protein conjugates (Supplemental Figure 6, A and B). Furthermore, adenovirus-mediated overexpression of IRF3 markedly suppressed UCP1 in human thermogenic adipocytes, an effect abolished by knockdown of HERC5 (Supplemental Figure 6, C and D).

Glycolytic enzymes are key ISG15 substrates in beige adipocytes. To elucidate the mechanism of action of ISG15, we used mass spectrometry-based proteomics to identify ISGylated proteins in an unbiased manner in primary beige adipocytes. Antibodies against ISG15 are ineffective in pulling down ISGylated proteins in other systems (33), and we found this to be true as well (not shown). Accordingly, we opted to use adenovirus to overexpress FLAG-ISG15 in Isg15 ${ }^{-/}$beige adipocytes treated with LPS, followed by pulldown with anti-FLAG (vs. IgG control) prior to mass spectrometry (Supplemental Figure 7A and Supplemental Table 2). Gene Ontology analysis of ISGylated proteins revealed a striking enrichment of several different metabolic pathways, including carbon metabolism, glycolysis/gluconeogenesis, glutathione metabolism, and purine metabolism (Figure 6A). Notably, virtually all glycolytic enzymes were identified as ISGylated by mass spectrometry (Figure 6, B and C), which was confirmed by immunoprecipitation followed by immunoblotting with anti-FLAG (Figure 6D). The effect of ISGylation on protein degradation is complex and target dependent (34). We used Western blotting to assess whether levels of glycolytic enzymes were altered in $I s g 15^{-/-}$beige adipocytes, but we noted no change in steady-state levels of any of the proteins tested (Supplemental Figure 7B).

We hypothesized that ISG15-mediated suppression of adaptive thermogenesis might occur through inhibition of glycolysis, which is essential for this process $(35,36)$. To assess this, we measured extracellular acidification rate (ECAR), a cellular index of glycolysis, in WT and Isg15 $15^{-/}$beige adipocytes in vitro. We found that loss of ISG15 was associated with elevated ECAR under both low- and high-glucose conditions (Figure 6E). Oligomycin treatment further increased ECAR in $\mathrm{Isg}_{\mathrm{g}} 5^{-/}$beige adipocytes, whereas blockage of glycolysis by 2-dexoy-D-glucose potently reduced ECAR of both WT and $I s g 15^{--}$adipocytes. Conversely, overexpression of ISG15 significantly decreased ECAR in $I s g 15^{-/-}$cells (Figure 6F). Similarly, $\operatorname{Irf} 3^{-/}$beige adipocytes exhibited elevated ECAR, which was fully suppressible by ISG15 overexpression (Figure 6G). Furthermore, overexpression of the de-ISGylase USP18 abolished the suppressive effect of IRF3 overexpression on ECAR (Figure 6H).

Consistent with these data, we found that $\mathrm{Isg} 15^{-/-}$beige adipocytes had significantly higher lactate levels than WT cells (Figure 
A

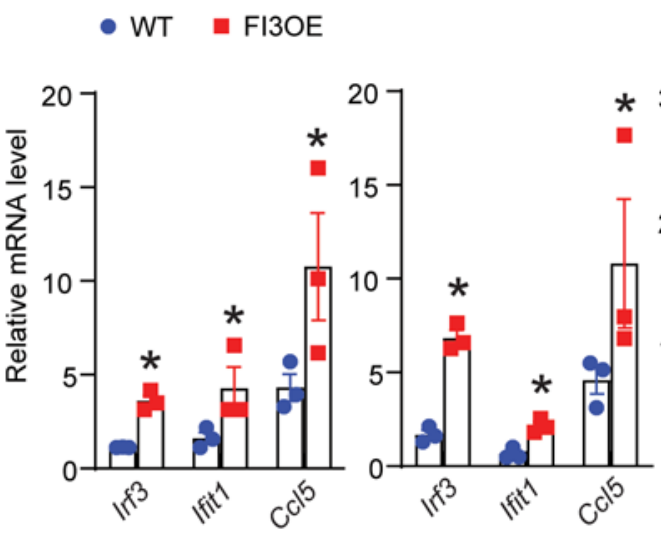

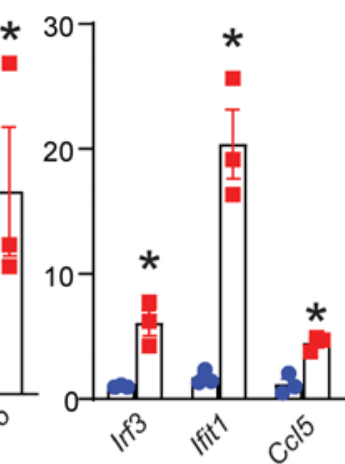

iWAT
D

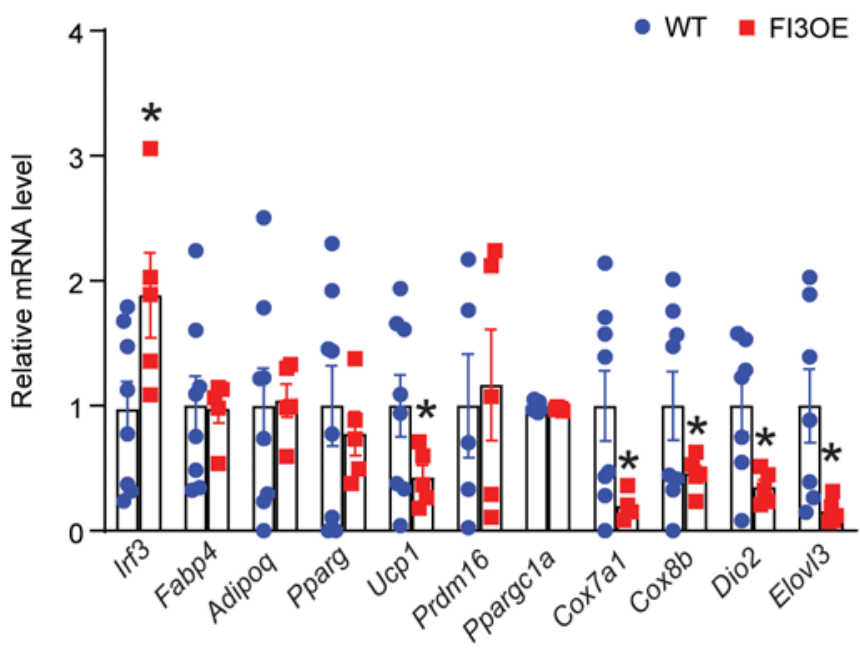

BAT

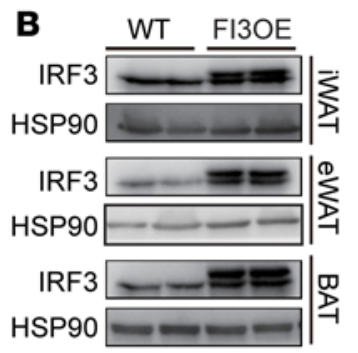

C

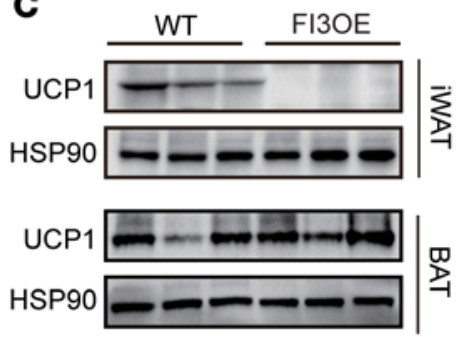

E
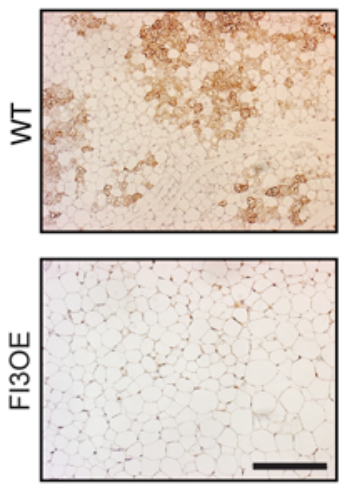

Figure 3. FI30E mice display decreased thermogenic gene expression. (A) Irf3, Ifit1, and CcI5 levels in iWAT, eWAT, and BAT of 8-week-old WT and FI30E mice $(n=5)$. (B) Western blot of IRF3 protein in iWAT, eWAT, and BAT of mice described in A. (C) Western blot of UCP1 in iWAT and BAT of chow-fed male WT and FI3OE mice after 7 days of cold challenge $(n=3)$. (D) Thermogenic gene expression in iWAT of mice described in C $(n=8-10)$. (E) UCP1 staining of iWAT of mice described in C. Scale bar: $100 \mu \mathrm{m}$. Statistical comparisons were made using 2-tailed Student's $t$ test $(\mathbf{A}$ and $\mathbf{D})$. Data are presented as mean \pm SEM. ${ }^{*} P<0.05$.

7A). We also employed metabolic flux analysis using $\left[\mathrm{U}^{13} \mathrm{C}\right] \mathrm{glu}-$ cose in these cells, demonstrating increased ${ }^{13} \mathrm{C}$ incorporation into glucose 6-phosphate (G6P) (M+6), pyruvate $(\mathrm{M}+3)$, and lactate $(\mathrm{M}+3)$ in the absence of ISG15 (Figure 7B). Moreover, $\mathrm{Irf3}^{-1}$ beige adipocytes show elevated lactate levels, which were normalized by overexpression of ISG15 (Figure 7C). Conversely, reduced lactate levels observed in FI3OE cells were rescued by overexpression of USP18 (Figure 7D). In order to maintain robust glycolysis during thermogenesis, a high cytosolic NAD level is essential for thermogenic adipocytes (37). Notably, the $\mathrm{NAD}^{+} /$ $\mathrm{NADH}$ ratio was also markedly higher in $\mathrm{Isg}^{\mathrm{S} 5^{-/}}$adipocytes (Figure 7E). Taken together, these findings indicate that ISG15 is a strong regulator of glycolysis in adipocytes.

ISGylation suppresses LDHA activity. We speculated that inhibition of lactate production might be the mechanism by which ISG15 represses thermogenesis. Consistent with this idea, treatment with the lactate dehydrogenase (LDH) inhibitor oxamate decreased

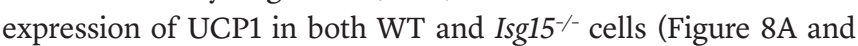

Supplemental Figure 7C). Conversely, the reduced UCP1 level observed in FI3OE cells was rescued by lactate treatment (Figure 8B and Supplemental Figure 7D).

Our proteomics data revealed one ISGylation site in LDHA (Supplemental Figure 7E), which converts pyruvate to lactate coupled with the recycling of $\mathrm{NAD}^{+}(38)$. This site is conserved in humans and mice (Figure 8C). To substantiate the association between LDHA and ISG15, we transfected hemagglutinin-tagged (HA-tagged) LDHA and FLAG-tagged ISG15 individually or together into HEK293T cells, followed by coimmunoprecipitation. As expected, HA-tagged LDHA was associated with FLAG-tagged ISG15 (Figure 8D and Supplemental Figure 7F), but not with FLAG-tagged ISG15AA, which lacks the $\mathrm{C}$-terminal diglycine required for ligation to the target lysine residue (Figure $8 \mathrm{E}$ ). We also determined that endogenous LDHA interacted with ISG15 in mouse primary beige adipocytes, an effect that was substantially enhanced by LPS treatment (Figure 8F). We next generated an LDHA mutant allele bearing a mutation at K57 (K57R) and tested whether it could be ISGylated. 
A

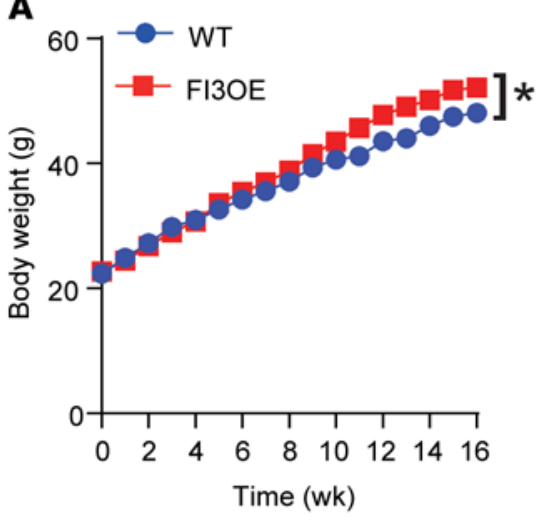

B

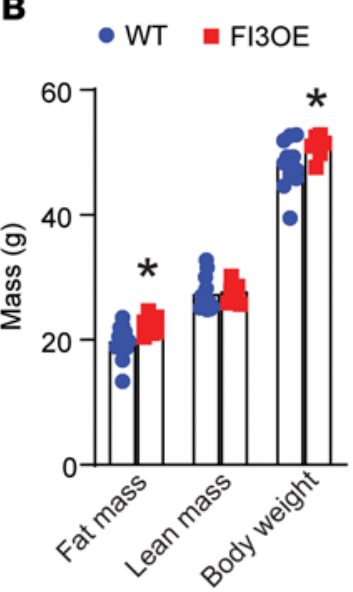

C

-WT $\because \mathrm{FI} 3 \mathrm{OE}$

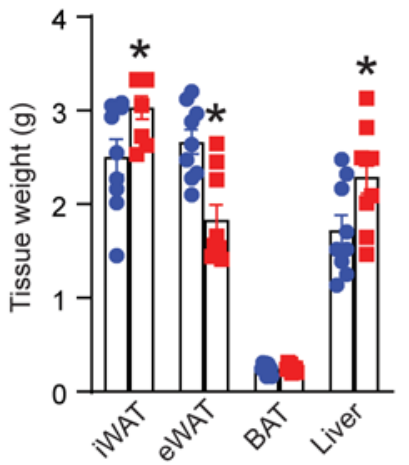

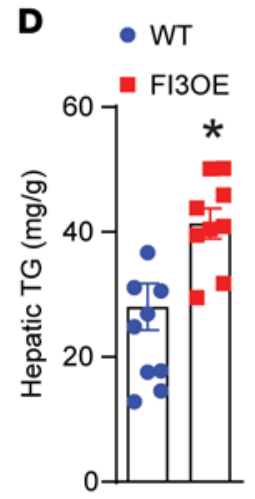

E
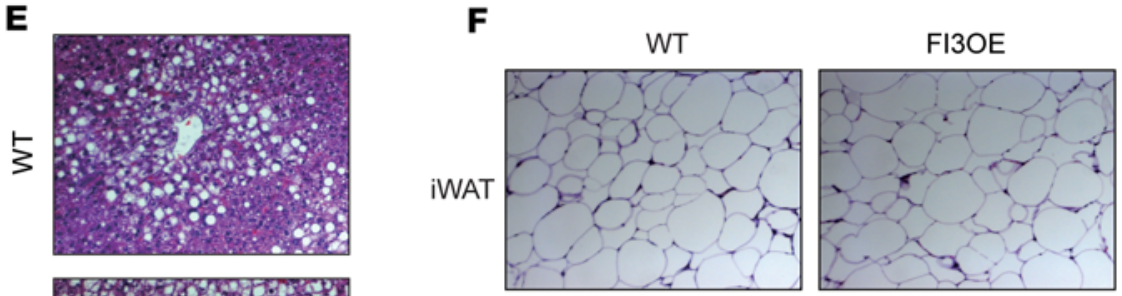

峁
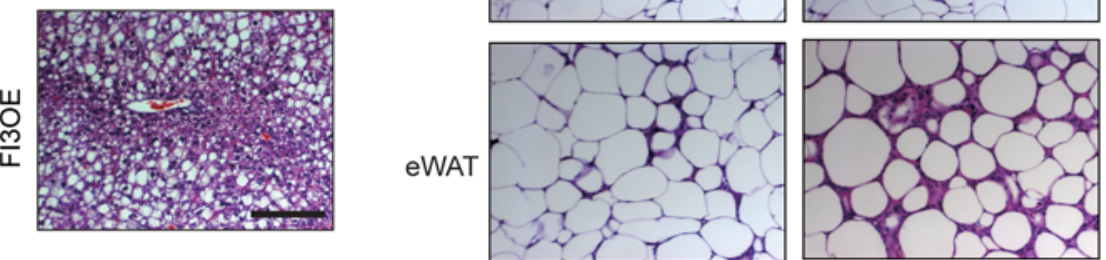

BAT
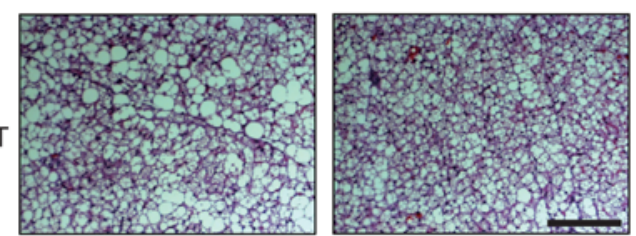

G AAC
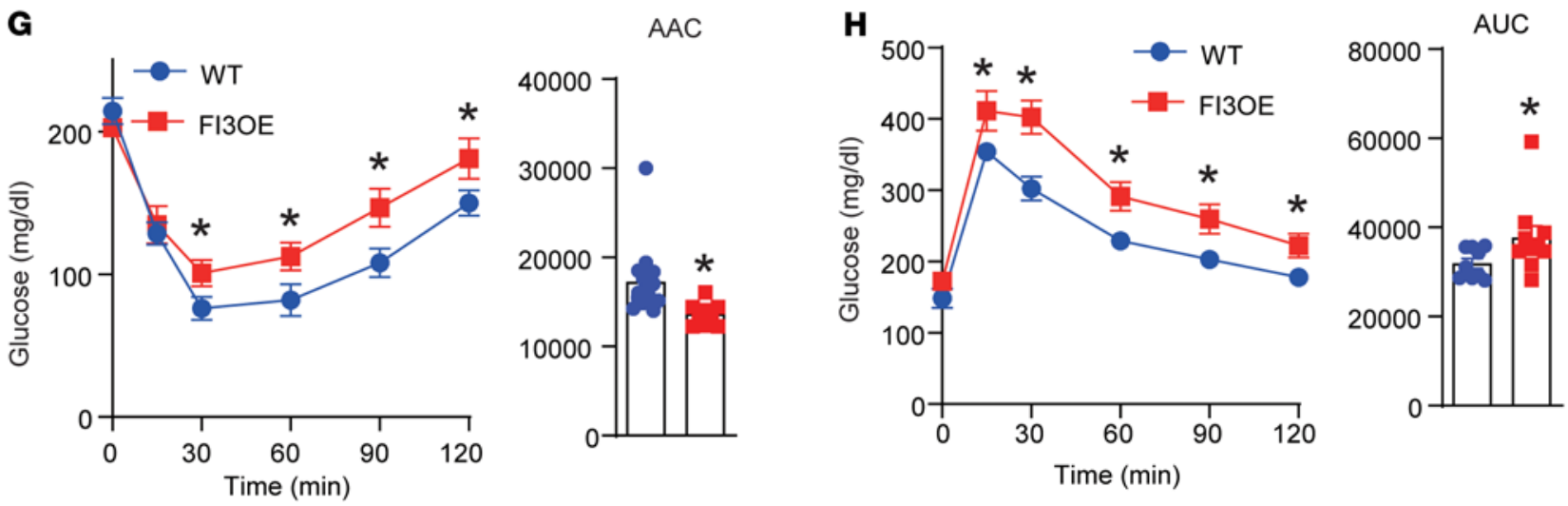

Figure 4. FI30E mice display increased diet-induced obesity. (A) Body weight of male WT and FI3OE mice during HFD feeding ( $n=8-10)$. (B) Body composition of male WT and FI3OE mice after 16 weeks of HFD feeding $(n=8-10)$. (C) Adipose depot and liver weight of male WT and FI30E mice after 16 weeks of high-fat feeding $(n=8-10)$. (D and E) Hepatic triglyceride (D) and H\&E staining of liver (E) of male WT and FI3OE mice after 16 weeks of HFD feeding ( $n=$ 8-10). (F) H\&E staining of adipose tissues of male WT and FI3OE mice after 16 weeks of high-fat feeding. Scale bars: $200 \mu \mathrm{m}$. (C) Insulin tolerance test (ITT) performed in male WT and FI3OE mice after 6 weeks on HFD. Right panel: Area above the curve of ITT ( $n=8-10)$. (H) Clucose tolerance test (GTT) performed in male WT and FI3OE mice after 6 weeks on HFD. Right panel: Area under the curve of GTT $(n=8-10)$. Statistical comparisons were made using 2-way ANOVA $(\mathbf{A}, \mathbf{G}$, and $\mathbf{H})$ or 2-tailed Student's $t$ test (B-D). Data are presented as mean \pm SEM. ${ }^{*} P<0.05$. 


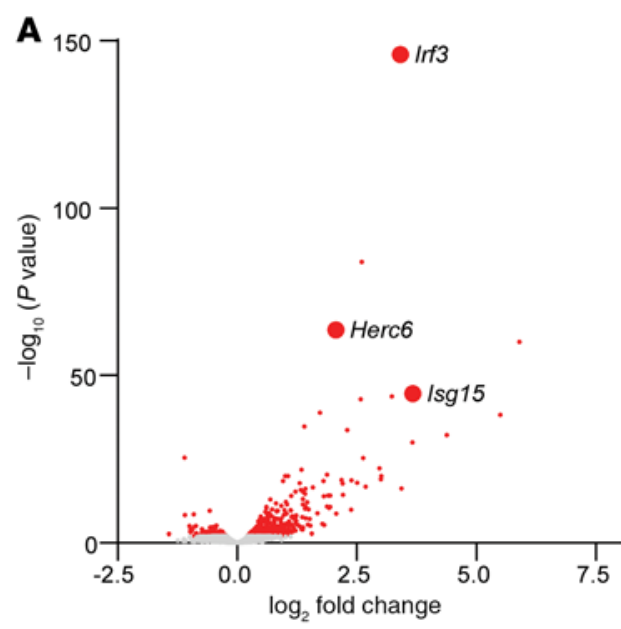

B

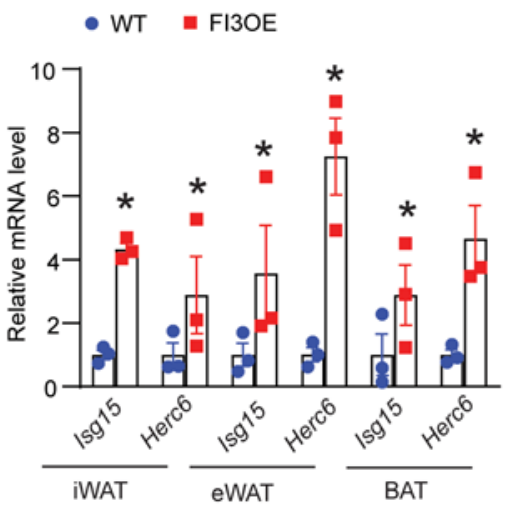

C

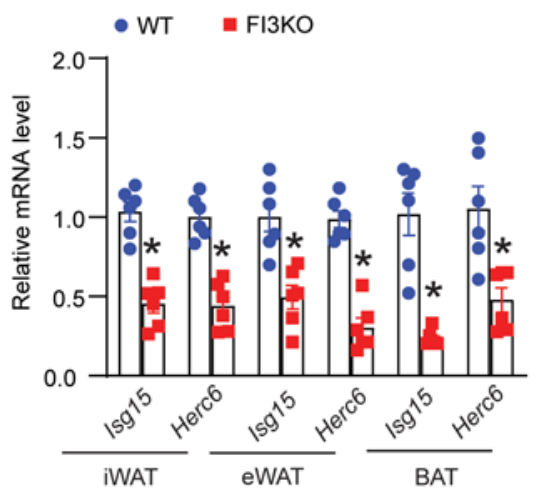

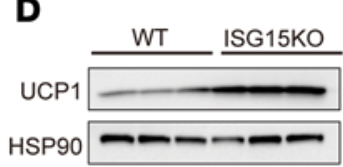

G

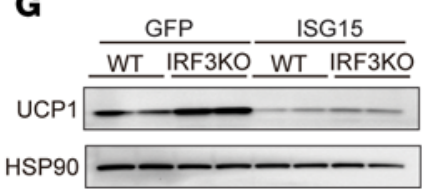

E

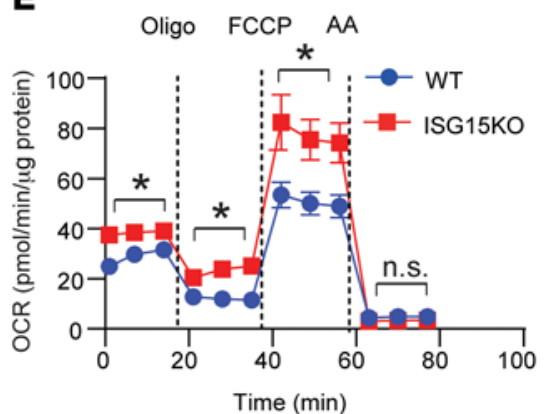

$\mathbf{F}$

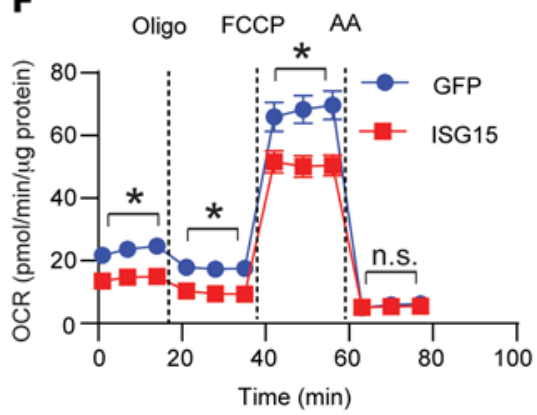

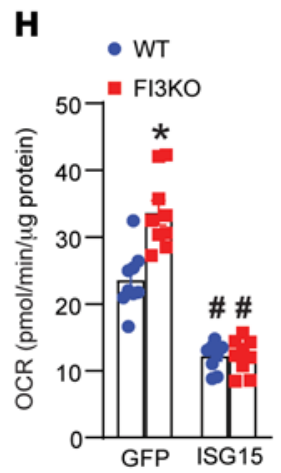

I

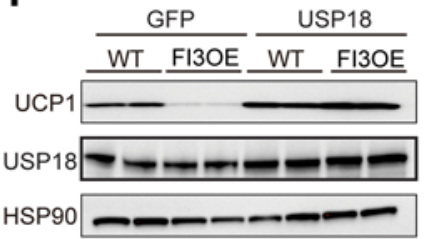

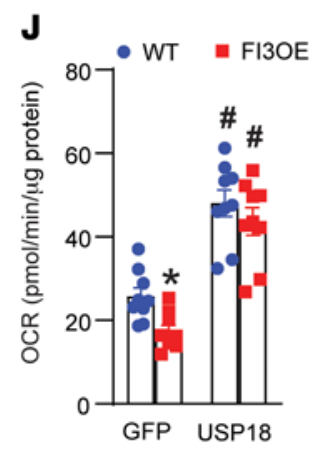

Figure 5. Adipocyte IRF3 suppresses UCP1 and mitochondrial activity through ISG15. (A) Volcano plot of RNA-Seq data from primary adipocytes isolated from iWAT of 12-week-old chow-fed male WT and FI3OE mice $(n=6)$. Genes in red are significantly different between WT and FI3OE groups. (B) mRNA levels of $I s g 15$ and Herc6 in primary adipocytes from iWAT, eWAT, and BAT depots in 12-week-old male chow-fed WT and FI30E mice $(n=6)$. (C) mRNA levels of Isg15 and Herc6 in primary adipocytes from iWAT, eWAT, and BAT in 12-week-old male chow-fed WT and FI3KO mice $(n=6)$. (D) Western blot of UCP1 in WT and Isg15 ${ }^{-1-}$ beige adipocytes $(n=3)$. (E) Oxygen consumption rate (OCR) of WT and Isg15-/- beige adipocytes $(n=8)$. FCCP, carbonyl cyanide-4-(trifluoromethoxy)phenylhydrazone; AA, antimycin A. (F) OCR in Isg15 GFP or murine ISG15. (H) Basal OCR in WT and Irf3 ${ }^{-/-}$beige adipocytes expressing GFP or murine ISC15 $(n=8)$. (I) Western blot of UCP1 and USP18 in WT and FI30E beige adipocytes expressing GFP or murine USP18. (J) Basal OCR in WT and FI3OE beige adipocytes expressing GFP or murine USP18 ( $n=8)$. Statistical comparisons were made using 2-way ANOVA (E and F) or 2-tailed Student's $t$ test (B, C, $\mathbf{H}$, and J). Data are presented as mean \pm SEM. ${ }^{*} P<0.05$; $P<0.05$ vs. GFP.

Loss of K57 completely blocked the interaction between ISG15 and LDHA (Figure 8G and Supplemental Figure 7G).

We next investigated how ISGylation affects the biochemical and functional properties of LDHA. Coimmunoprecipitation experiments showed that ubiquitination of LDHA was not affected by ISGylation (Supplemental Figure 7H). However, LDHA activity was markedly suppressed by ISGylation, an effect abolished in the K57R LDHA mutant (Figure $8 \mathrm{H}$ ). Similarly, the induction of cellular lactate by LDHA overexpression was significantly repressed by ISG15, which was also blocked by the K57R mutant (Figure 8I). These results indicate that ISG15 suppresses LDHA activity via covalent modification of Lys57.

ISG15 represses thermogenesis in vivo. To directly assess the role of ISG15 in adaptive thermogenesis in vivo, we performed an acute cold tolerance test and found that Isg15 ${ }^{-/-}$mice were notably more cold-resistant than WT mice (Figure 9A). Isg15 ${ }^{-/}$mice also showed increased expression of UCP1 and other thermogenic markers in both iWAT and BAT after chronic cold challenge (Figure 9, B-D, and Supplemental Figure 8A). Consistent with these findings, Isg15 $15^{-/-}$mice had 
A

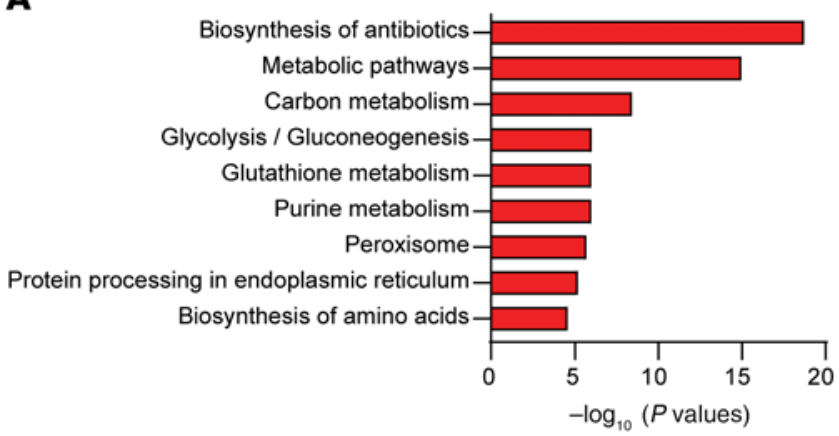

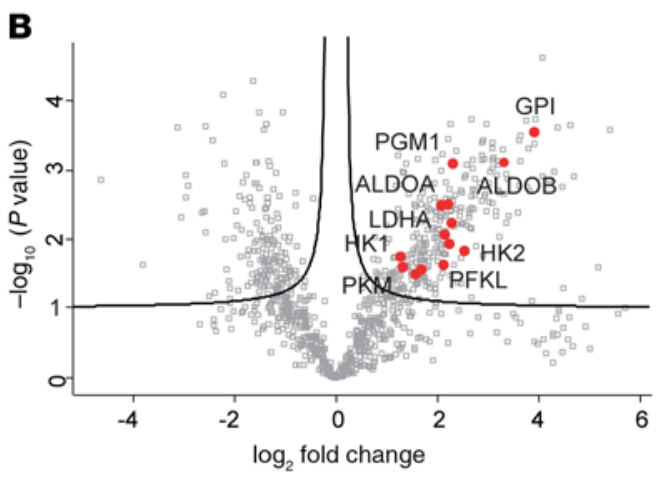

D
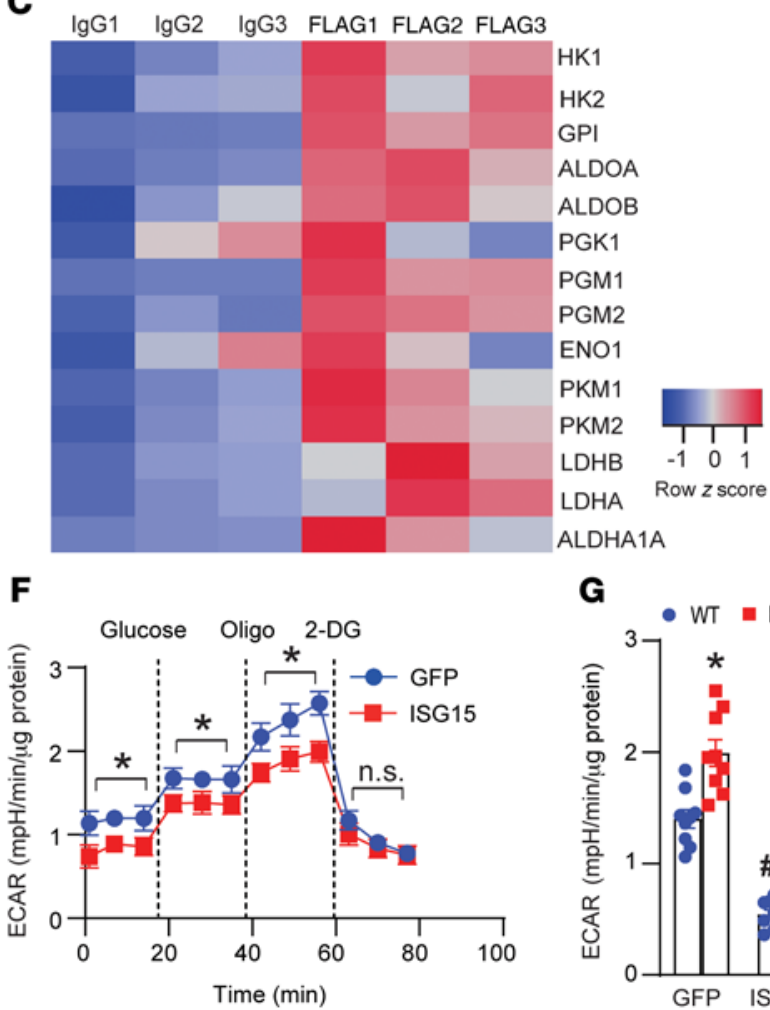

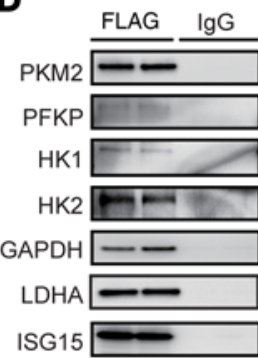

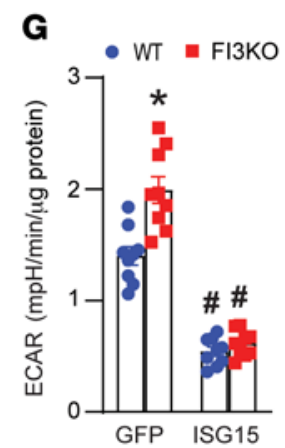

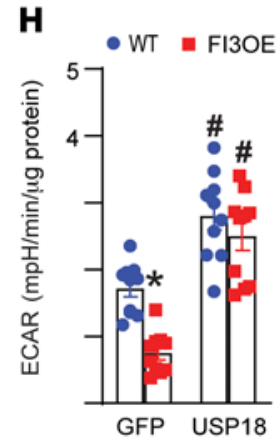

E

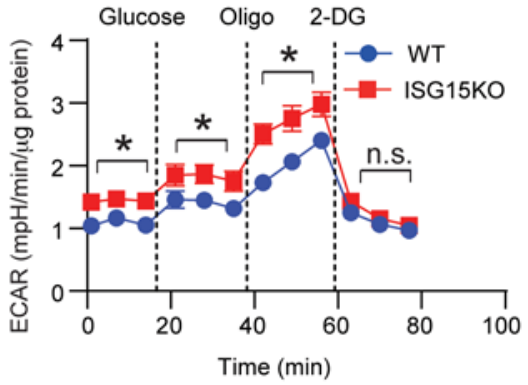

Figure 6. Glycolytic enzymes are key ISG15 substrates in beige adipocytes. (A) Gene Ontology analysis (biological process) of ISGylated proteins ( $n=3$ ). (B) Volcano plot of proteomic analysis. Red dots represent glycolytic enzymes. Black lines indicate significantly enriched or de-enriched proteins (FDR $=0.05)$ ( $n$ = 3). (C) Heatmap of glycolytic protein levels from mass spectrometric analysis $(n=3)$. (D) Western blot of glycolytic enzymes in beige adipocytes expressing GFP or FLAG-ISG15 pulled down by IgG or anti-FLAG antibody. (E) Extracellular acidification rate (ECAR) of WT and $I s g 15^{-/-}$beige adipocytes ( $n=8$ ). 2-DG, 2-dexoy-D-glucose. (F) ECAR in Isg15 adipocytes expressing GFP or mouse ISC15 $(n=8)$. (H) Basal ECAR in WT and FI3OE beige adipocytes expressing GFP or mouse ISC15 $(n=8)$. Statistical comparisons were made using 2-way ANOVA (E and $\mathbf{F}$ ) or 2-tailed Student's $t$ test (G and $\mathbf{H})$. Data are presented as mean \pm SEM. ${ }^{*} P<0.05 ;{ }^{\#} P<0.05$ vs. WT.

increased numbers of multilocular UCP1+ cells in iWAT (Figure 9E). Similarly, CL316,243 treatment increased expression of thermogenic markers in iWAT and BAT of WT mice, but had a greater effect in Isg15 $\%$ mice (Supplemental Figure 8, B and C). Furthermore, indirect calorimetry showed that $\mathrm{Isg}_{15}{ }^{-}$mice had higher energy expenditure and oxygen consumption at both room temperature and $4^{\circ} \mathrm{C}$. However, at thermoneutrality, no differences in oxygen consumption or energy expenditure were observed (Figure 9, F and G). These results demonstrate that ISG15 depletion increases adaptive thermogenesis in adipose tissue and enhances whole-body energy expenditure.

Because ISG15 has been reported to exert effects that are independent of ISGylation (34), we generated adipocyte-specific
Herc6-knockout mice using a CRISPR/Cas9-based approach (39). We crossed Cre-dependent Rosa26-Cas9 knockin mice (Rosa26-LSL-Cas9) with FI3OE mice to generate FI3OE/Cas9 and Cas9-only mice (Figure 10A). All mice then received bilateral intrainguinal WAT injections of AAV2/8 expressing either a specific guide RNA targeting exon 1 of the Herc 6 gene, thus specifically deleting Herc6 in inguinal adipocytes, or an empty control vector (Figure 10B). In FI3OE/Cas9 mice, deletion of Herc6 profoundly reduced the ability of the IRF3-2D allele to suppress thermogenic gene expression after chronic cold challenge (Figure 10, C and D). These data indicate that ISGylation is required for IRF3-mediated suppression of adaptive thermogenesis. 
A

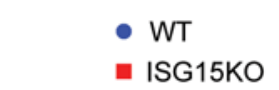

B

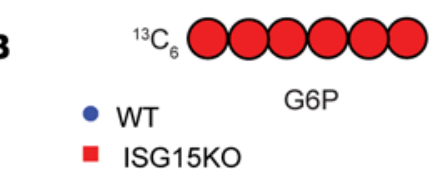

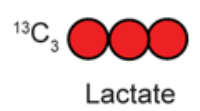

Lactate

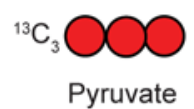

Pyruvate
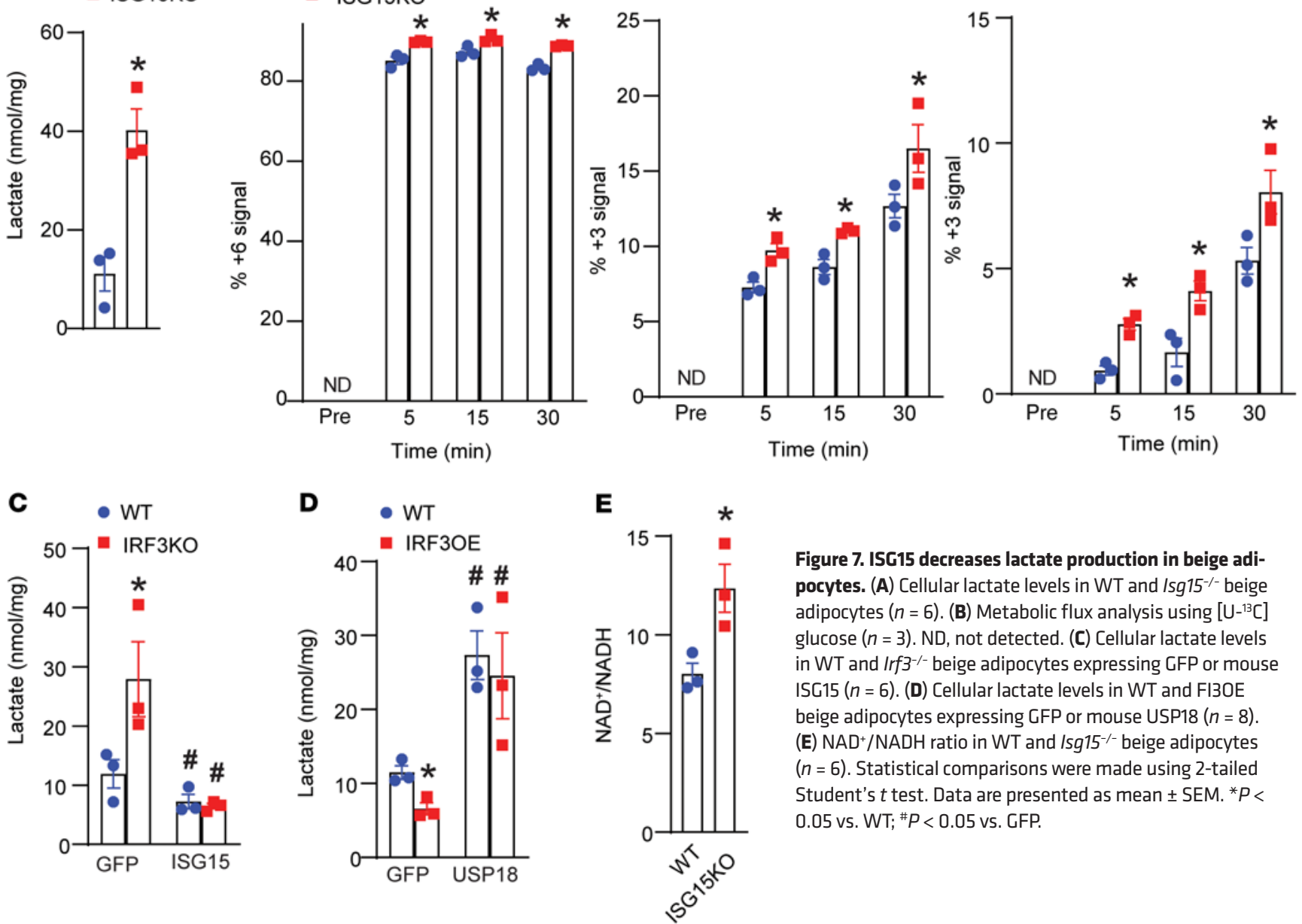

Figure 7. ISC15 decreases lactate production in beige adipocytes. (A) Cellular lactate levels in WT and $/ \mathrm{sg} 15^{-1-}$ beige adipocytes $(n=6)$. (B) Metabolic flux analysis using [U- $\left.{ }^{13} \mathrm{C}\right]$ glucose $(n=3)$. ND, not detected. (C) Cellular lactate levels in WT and $\mathrm{Irf3} 3^{-1-}$ beige adipocytes expressing CFP or mouse ISG15 $(n=6)$. (D) Cellular lactate levels in WT and FI3OE beige adipocytes expressing GFP or mouse USP18 $(n=8)$. (E) NAD /NADH ratio in WT and $/ s g 15^{-/-}$beige adipocytes $(n=6)$. Statistical comparisons were made using 2-tailed Student's $t$ test. Data are presented as mean \pm SEM. ${ }^{*} P<$ 0.05 vs. WT; ${ }^{P}<0.05$ vs. GFP.

ISG15 is correlated with insulin sensitivity and glucose homeostasis in mice and humans. To address the clinical relevance of our findings, we sought to determine whether ISG15 expression is elevated in the adipose tissue of obese humans. In subcutaneous fat, ISG15 mRNA levels were significantly correlated with increased BMI (Figure 11A). We also found that Isg15 mRNA levels were increased in adipocytes, but not SVF, of murine iWAT and eWAT (Supplemental Figure 8D). HFD markedly induced levels of both free ISG15 and ISGylated protein conjugates in primary adipocytes of iWAT from WT mice (Figure 11B and Supplemental Figure 8E). Isg15 $\%$ mice showed no significant differences in body weight or adiposity on chow diet (Supplemental Figure 8, F and G). Similarly, no differences in insulin tolerance and glucose tolerance were detected on a chow diet (Supplemental Figure 8, H and I).

On HFD, Isg15 mice (Figure 11C). Body composition analysis revealed that Isg15 mice had lower fat mass than WT mice, with comparable lean mass (Figure 11D). Liver, BAT, perirenal WAT, and iWAT weights were all markedly reduced in knockout mice, whereas eWAT weight was not (Figure 11, E and F, and Supplemental Figure 8J). Lipid accumulation in the liver was also lower in knockout mice (Figure 11, $\mathrm{G}$ and $\mathrm{H})$. Knockout mice exhibited increased insulin and glucose tolerance after 16 weeks of HFD feeding (Figure 11, I and J), with significantly lower fed and fasting plasma insulin levels (Figure $11 \mathrm{~K})$. These results indicate that $I s g 15^{-\digamma}$ mice are more resistant to diet-induced obesity and glucose intolerance than WT mice, thus phenocopying the FI3KO mice.

To assess whether the effects of ISG15 ablation on body weight and glucose homeostasis are dependent on altered body weight, we challenged $\mathrm{Isg}_{5} 15^{--}$and WT mice with HFD at thermoneutrality for 16 weeks. Under these conditions, Isg15 ${ }^{--}$mice showed no difference in body weight adiposity or food intake compared with WT mice on HFD (Supplemental Figure 9, A-C). Consistently, we found no differences in tissue weight between Isg1 $15^{--}$and WT mice (Supplemental Figure 9D). HFD-fed $I s g 15^{-/}$mice at $30^{\circ} \mathrm{C}$ also showed insulin tolerance and glucose tolerance similar to those in WT mice (Supplemental Figure 9, E and F), with comparable fed and fasting plasma insulin levels (Supplemental Figure $9 \mathrm{G})$. Therefore, the metabolic benefits observed in $\mathrm{Isg}_{\mathrm{s}} 15^{-/}$mice on HFD accrue mainly through increased adaptive thermogenesis.

\section{Discussion}

The conversion of energy into heat by specialized adipocytes is a critical mechanism by which mammals maintain body temperature, 
A

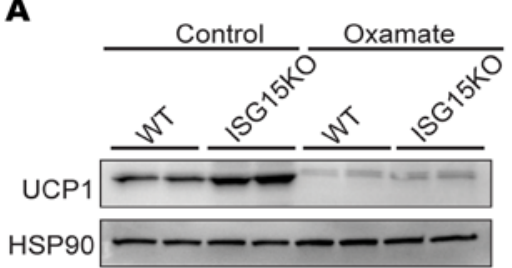

B

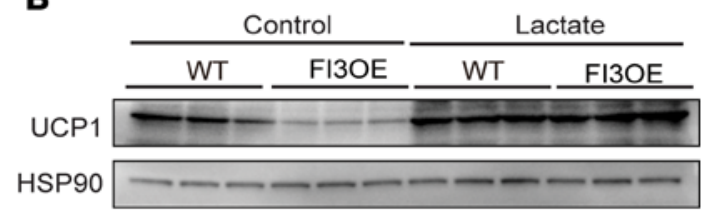

C

Human 45 ADELALVDV I EDKLKG 60 Mouse 74 ADELALVDVMEDKLKG 89
D

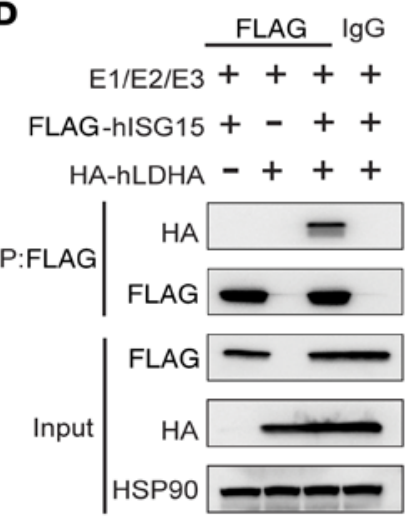

G
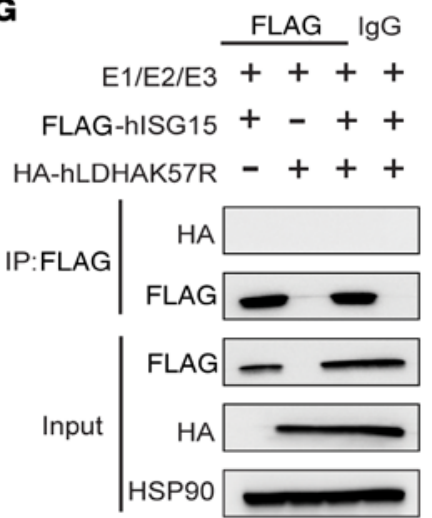

I

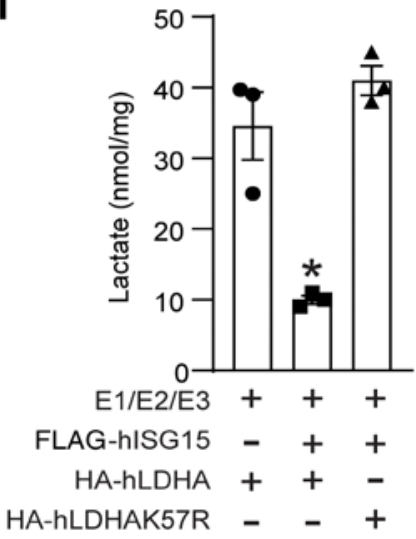

E

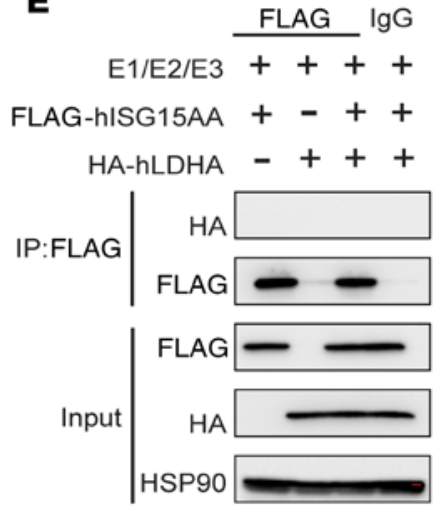

H

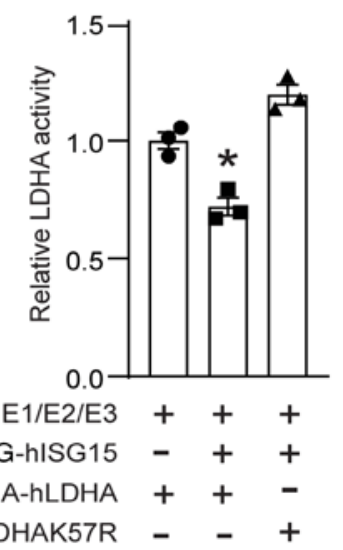

F
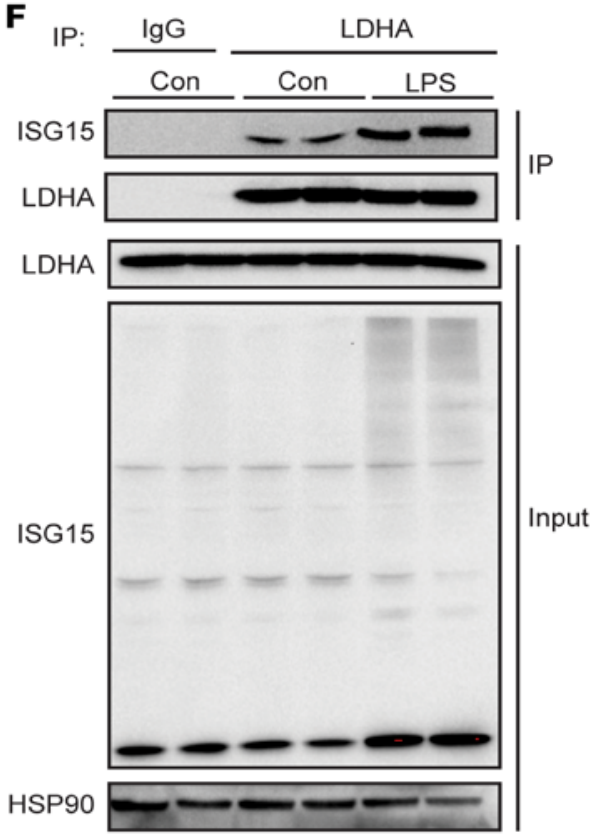

Figure 8. ISG15 decreases lactate production by suppressing LDHA activity. (A) Western blot of UCP1 in WT and $/ \mathrm{sg} 15^{-/-}$beige adipocytes treated with control or $50 \mathrm{mM}$ oxamate. (B) Western blot of UCP1 in WT and $/ \mathrm{sg} 15^{-/-}$beige adipocytes treated with control or $10 \mathrm{mM}$ lactate. (C) Alignment of conserved ISGylation site in LDHA protein sequences of different species. (D, E, and G) HEK293T cells were transfected with the indicated plasmids. Twenty-four hours after transfection, cell lysates were immunoprecipitated with an anti-FLAG antibody and then immunoblotted with the indicated antibodies. (F) Endogenous coimmunoprecipitation of LDHA and ISG15 in mouse primary beige adipocytes treated with control or LPS $(100 \mathrm{ng} / \mathrm{mL}$ ) for 12 hours. (H and I) HEK293T cells were transfected with the indicated plasmids. Twenty-four hours after transfection, cellular LDHA activity $(\mathbf{H})$ and cellular lactate (I) were measured $(n=3)$. Statistical comparisons were made using 2-tailed Student's $t$ test. Data are presented as mean \pm SEM. ${ }^{*} P<0.05$.

and also has significant implications for energy homeostasis. There is abundant evidence from mouse models that reduced thermogenesis promotes excess weight gain in the setting of HFD (40-42). In humans, there is an inverse association between adiposity and the activity of brown fat $(6,43,44)$. In addition, some human obesity GWAS genes may act by reducing adipose thermogenesis (45), although it remains uncertain whether defects in adipose thermo- genesis play a major instigating role in human obesity. Nonetheless, it is clear that as obesity develops, energy expenditure fails to compensate for the excess calories ingested; accordingly, understanding the mechanisms by which adipose thermogenesis becomes restrained in overnutrition could open new horizons in the management of obesity.

Obesity induces a state of chronic inflammation in adipose tissue, with a shift in the immune cell composition of the fat pad and 


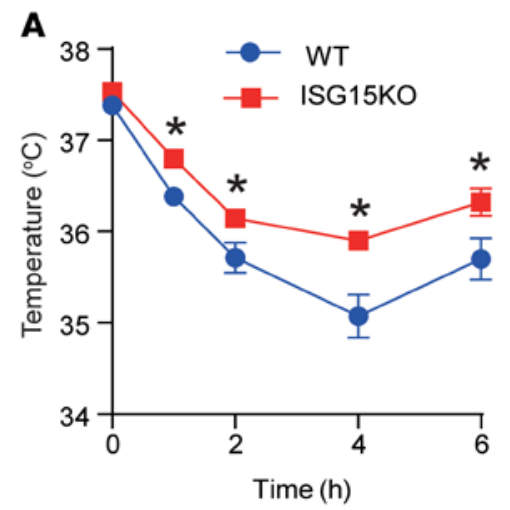

B

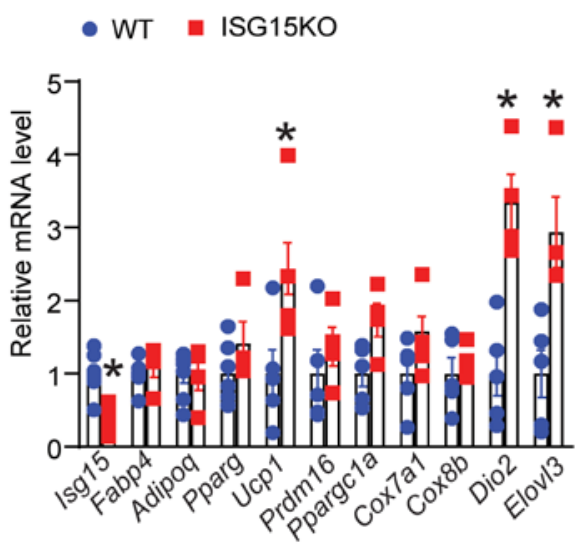

C

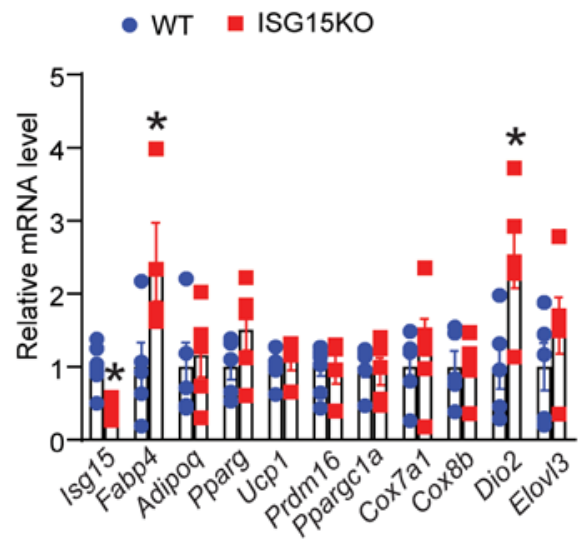

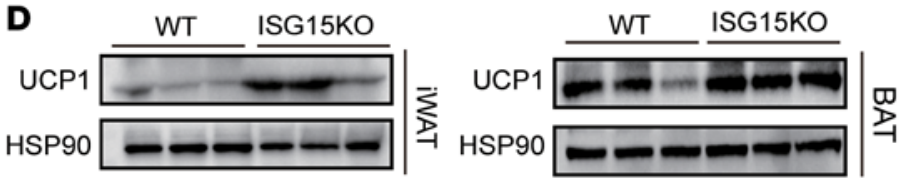

F $\quad$ WT $=$ ISG15KO $23^{\circ} \mathrm{C}$

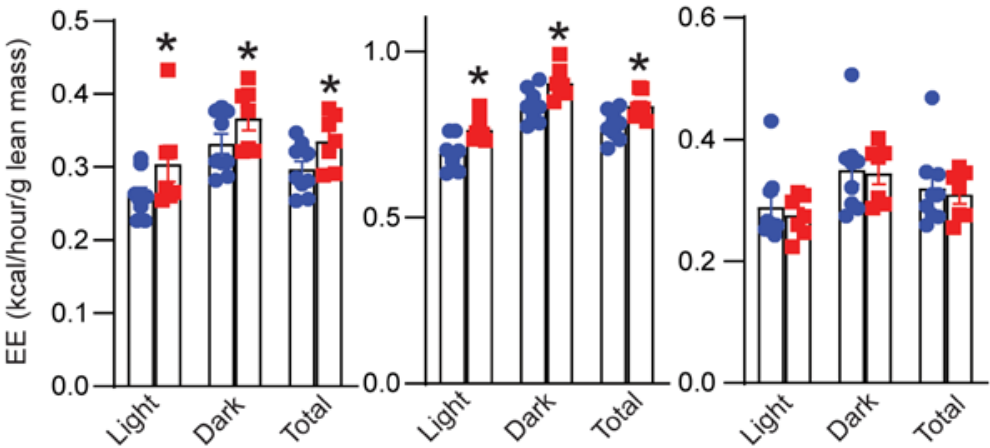

G

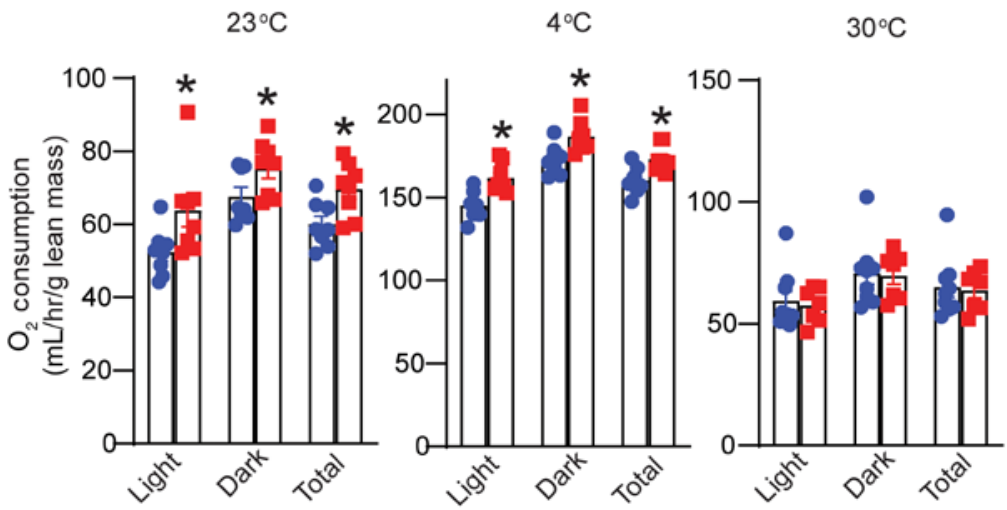

E

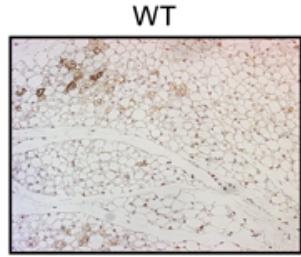

ISG15KO

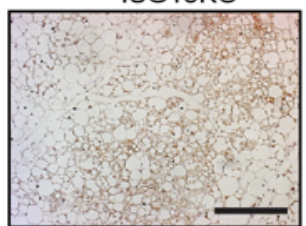

Figure 9. Isg15 $1 /$ mice display increased thermogenesis. (A) Acute cold tolerance test of 12-week-old male WT and $1 \mathrm{sg} 15^{-/-}$mice $(n=10)$. (B) Thermogenic gene expression in iWAT of 12-week-old male chow-fed WT and Isg15 $15^{-/-}$mice after 7 days of cold challenge ( $n$ = 8-10). (C) Thermogenic gene expression in BAT of 12-week-old male chow-fed WT and $1 \mathrm{sg} 15^{-/-}$mice after 7 days of cold challenge (n = 8-10). (D) Western blot of UCP1 in iWAT and BAT of 12-week-old male chow-fed WT and Is $15^{-/-}$mice after 7 days of cold challenge. (E) UCP1 staining in iWAT of 12-week-old male chow-fed WT and $/ \mathrm{sg} 15^{-1-}$ mice after 7 days of cold challenge. Scale bar: $100 \mu \mathrm{m}$. (F) Energy expenditure (EE) of 12-week-old male chowfed WT and $/ \mathrm{sg} 15^{-/-}$mice by indirect calorimetry $(n=8)$. (G) Oxygen consumption rate of 12-week-old male chow-fed WT and $/ \mathrm{sg} 15^{-1-}$ mice by indirect calorimetry $(n=8)$. Statistical comparisons were made using 2-way ANOVA (A) or 2-tailed Student's $t$ test (B, C, F, and G). Data are presented as mean \pm SEM. ${ }^{*} P<0.05$. of a wide array of cytokines; this promotes insulin resistance and other metabolic sequelae. While this has been studied primarily in WAT, a similar phenomenon occurs in BAT $(14,46)$. The resulting proinflammatory milieu has strong antithermogenic effects, including repression of $U c p 1$ expression in brown fat, and the inhibition of beige adipogenesis $(9,11,13,14,46)$. It seems likely, therefore, that inflammation in brown and beige adipose tissues contributes signifi- cantly to inadequate compensation of adipose thermogenesis during obesity, although the mechanism is unclear.

Proinflammatory signaling molecules, including TNF- $\alpha$, TLR4 ligands, IKKe, and JNK1, have been linked to suppression of adipose thermogenesis $(12,47-50)$. Most of these signals converge on $\mathrm{NF}-\kappa \mathrm{B}$; thus NF- $\kappa \mathrm{B}$ activation has been suggested to play a major role in the transcriptional effects of inflammation in adipose tissue. 
A

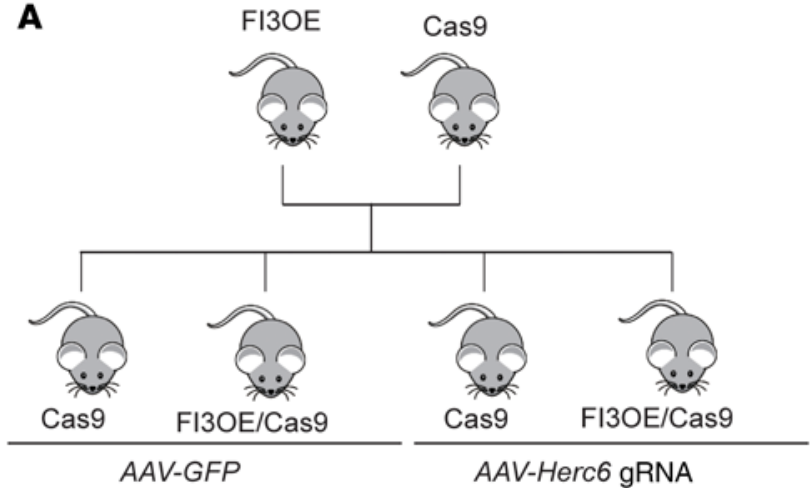

C

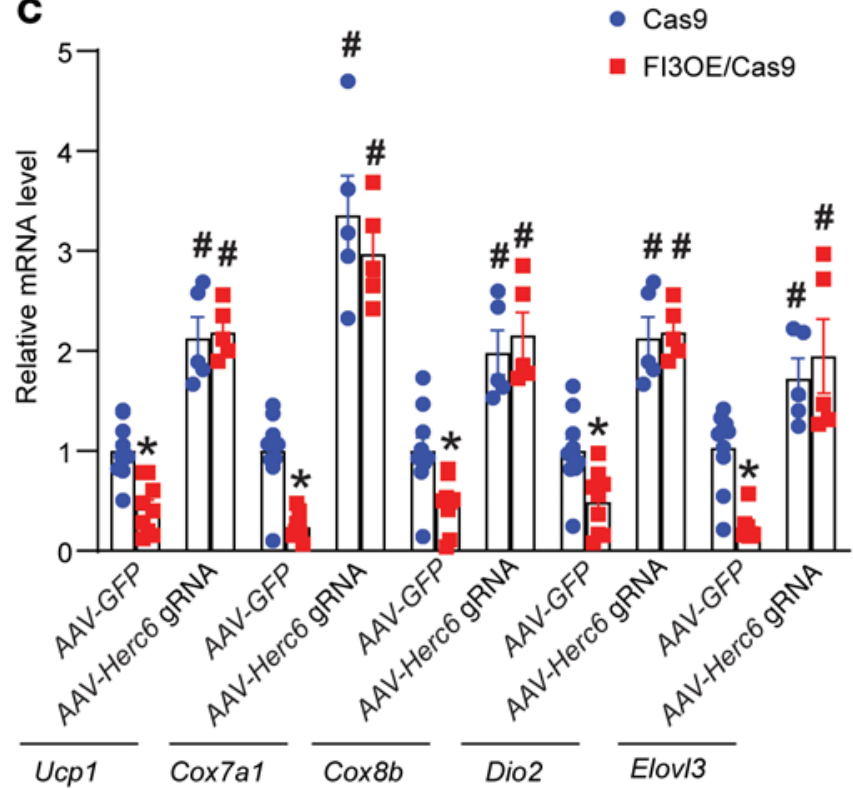

B - AAV-GFP = AAV-Herc6

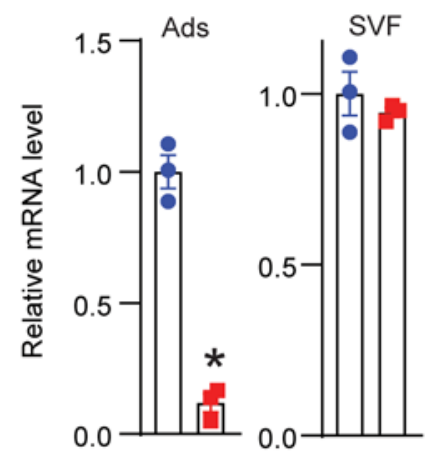

D
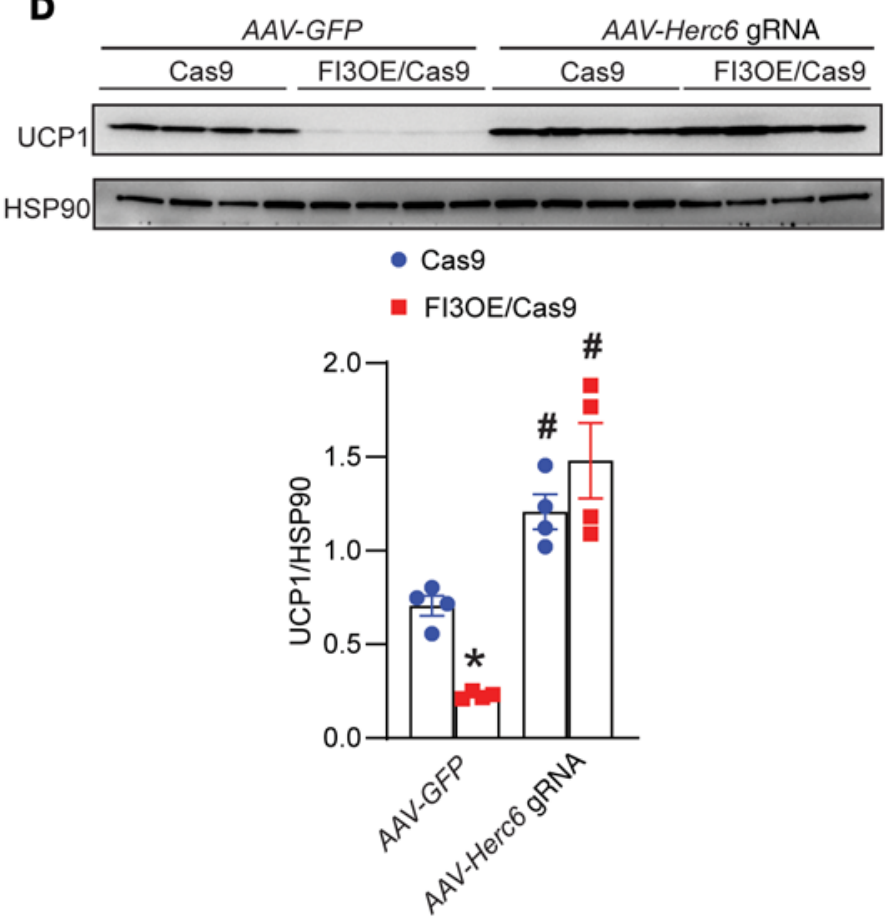

Figure 10. Herc6 is required for adipocyte IRF3-mediated suppression of thermogenesis. (A) Schematic illustrating the strategy for the generation of Fl30E/Cas9 mice. (B) mRNA levels of Herc6 in primary adipocytes and SVF from iWAT of Adipoq-Cre/Cas9 mice injected with AAV-GFP or AAV-Herc6 guide RNA (gRNA) ( $n=$ 4). (C) Thermogenic gene expression in iWAT of Cas9 and FI3OE/Cas9 mice injected with AAV-GFP or AAV-Herc6 gRNA followed by 7 days of cold challenge ( $n=6$ ). (D) Western blot of UCP1 in iWAT of mice as described in C $(n=4)$. Statistical comparisons were made using 2-way ANOVA (C and $\mathbf{D})$ or 2-tailed Student's $t$ test (B). Data are presented as mean \pm SEM. ${ }^{*} P<0.05$ vs. WT; ${ }^{*} P<0.05$ vs. AAV-GFP.

Data directly implicating NF- $\mathrm{BB}$ in this process, though, are conflicting. Mice with loss of $\mathrm{p} 65$, the active subunit of $\mathrm{NF}-\kappa \mathrm{B}$, in adipocytes and macrophages are leaner than controls on an HFD, with increased energy expenditure (16). However, mice in which p65 has been activated in these same cell types, either by transgenic overexpression or by targeted ablation of the p50 regulatory subunit, are unexpectedly also resistant to diet-induced obesity (17). In addition, IL-6 is a major effector of NF- $\mathrm{kB}$ activity, yet it has inconsistent effects on $U c p 1$ expression and energy expenditure (51); in fact, BAT is the dominant source of serum IL- 6 in the mouse (52). IRF3 is another proinflammatory transcription factor, typically associated with the type I interferon response in immune cells. We have shown that global $\mathrm{Irf3}^{-/-}$mice are resistant to diet-induced obesity as a result of increased browning and energy expenditure (23).
IRF3 is activated by 2 types of pattern recognition receptors, one involving Toll-like receptors (in particular, TLR3 and TLR4) and the other mediated by the cGAS/STING pathway, which is responsive to double-stranded DNA (53). These upstream receptors converge on kinases that phosphorylate IRF3, causing dimerization and nuclear translocation (54). Interestingly, all 3 known IRF3 upstream kinases, inhibitor of NF- $\kappa \mathrm{B}$ kinase subunit $\varepsilon(\mathrm{IKK} \varepsilon)$, TANK-binding kinase 1 (TBK1), and apoptosis signal-regulating kinase 1 (ASK1), have been shown to inhibit adipose thermogenesis in WAT and/ or BAT $(49,55,56)$. Several mechanisms have been proposed to account for these effects. For example, IKK $\varepsilon$ and TBK1 activate phosphodiesterase 3B, inducing resistance to catecholamines with subsequent reduction in lipolysis and energy expenditure (49). TBK1 also directly phosphorylates and inhibits AMP kinase (AMPK) 
A

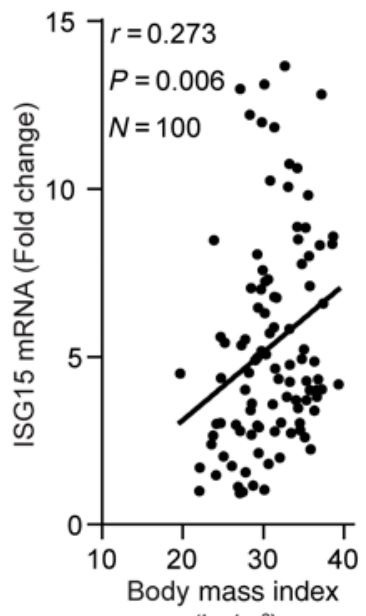

$\left(\mathrm{kg} / \mathrm{m}^{2}\right)$

E

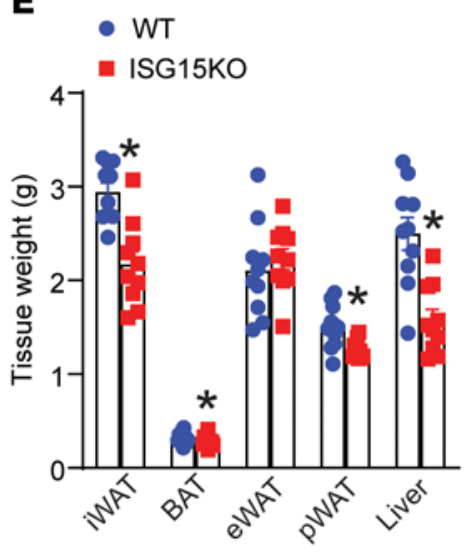

$\mathbf{F}$
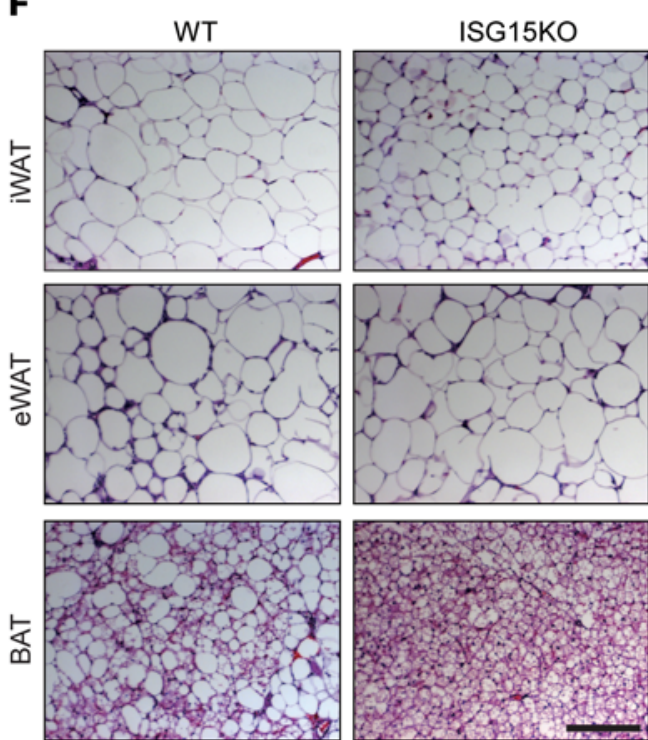

G

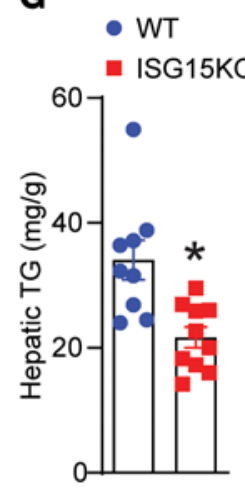

H

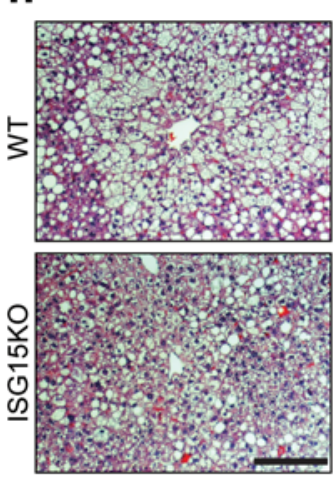

C

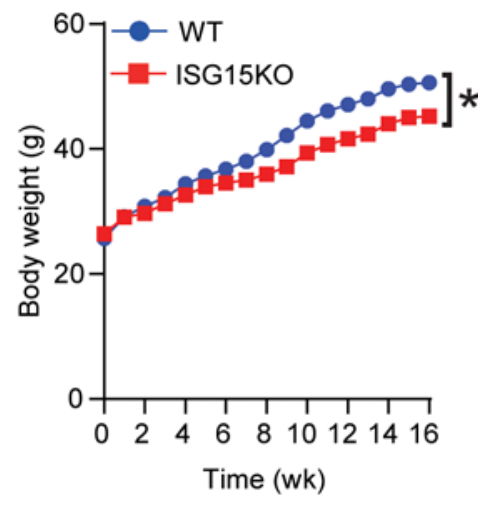

D

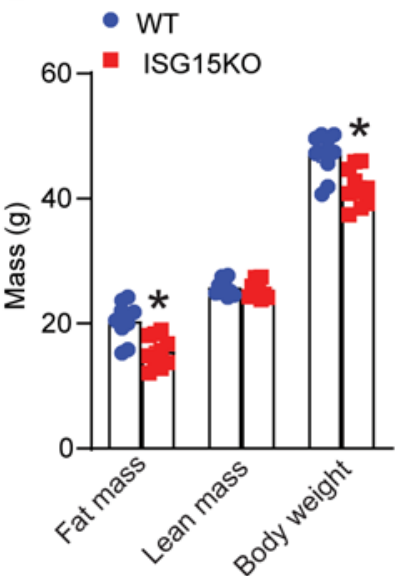

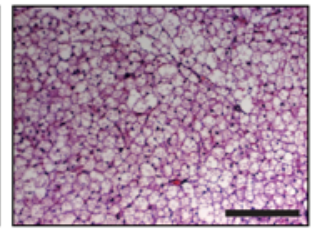

I

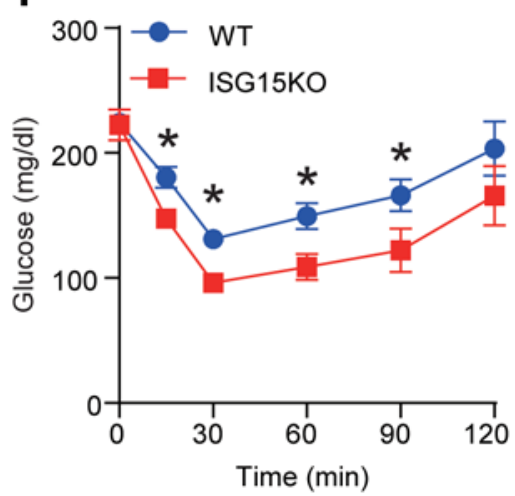

AUC

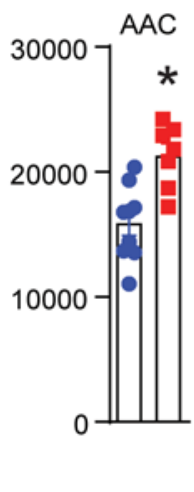

$\mathbf{K}$

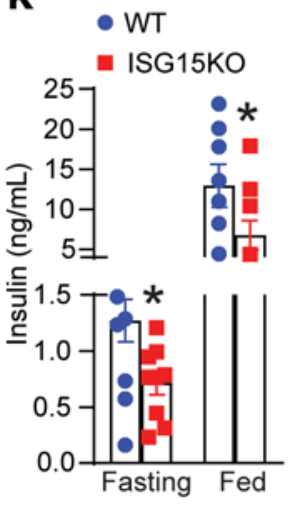

Figure 11. ISG15 is positively correlated with insulin sensitivity and glucose homeostasis. (A) Isg15 mRNA expression correlates with BMI in human subjects. (B) Western blot of free ISG15 and ISC15 conjugates in primary adipocytes from chow- and HFD-fed mice. (C) Body weight of male WT and Isg15 ${ }^{-/}$ mice during high-fat feeding $(n=8-10)$. (D) Body composition of male WT and $/ s g 15^{-1-}$ mice after 16 weeks of HFD feeding ( $\left.n=8-10\right)$. (E) Adipose depot and liver weight of male WT and $I s g 15^{-1-}$ mice after 16 weeks of HFD feeding $(n=8-10)$. (F) H\&E staining of adipose tissues of male WT and $/ s g 15^{-/-}$mice after 16 weeks of HFD feeding. Scale bar: $200 \mu \mathrm{m}$. (C) Hepatic triglyceride content of male WT and $/ \mathrm{sg} 15^{-1-}$ mice after 16 weeks of HFD feeding ( $\left.n=8-10\right)$. (H) H\&E staining of liver of male WT and $/ \mathrm{sg} 15^{-/-}$mice after 16 weeks of HFD feeding. Scale bar: $200 \mu \mathrm{m}$. (I) Insulin tolerance test (ITT) performed in male WT and $I s g 15^{-1-}$ mice after 16 weeks of HFD feeding $(n=8-10)$. Right panel: Area above the curve of ITT $(n=8-10)$. (J) Glucose tolerance test (GTT) performed in mice as described as $\mathbf{D}$. Right panel: Area under the curve of GTT ( $n=8-10)$. (K) Fed and fasting plasma insulin levels in mice as described as $\mathbf{D}$ ( $n=8-10)$. Statistical comparisons were made using 2-way ANOVA (C, I, and J) or 2-tailed Student's $t$ test (D, E, and K). Data are presented as mean \pm SEM. ${ }^{*} P<0.05$. 
in adipose tissue, resulting in reduced lipid oxidation and thermogenesis (57). Although our study did not focus on the upstream activators of IRF3, these prior results are consistent with the notion that activation of IRF3 (and its target ISG15) represents a key component of the antithermogenic actions of inflammatory signaling.

IRF3 is generally thought of as a transcriptional effector of immune cells, but it is widely expressed throughout the body. There is, however, little appreciation of its actions in nonimmune cells. In our prior work we showed that IRF3 protein levels increase in isolated adipocytes in obesity (23). Studies in cultured adipocytes suggested that IRF3 acts to suppress thermogenic gene expression and to promote insulin resistance. These phenotypes were recapitulated in $\mathrm{Irf3}^{-/-}$mice in vivo. A major caveat of these experiments is that it is hard to definitively ascribe the phenotype to intra-adipocyte IRF3 in a global knockout model. Furthermore, $\operatorname{Irf} 3^{-/-}$mice are known to carry an inadvertent knockout of a nearby gene, $B c l 2 l 12$, which also has important immune functions (26). To circumvent these issues, we developed $\operatorname{Irf} 3^{f l o x}$ mice, which enable the study of IRF3 function in a cell type-specific fashion. These mice have normal Bcl2l12 expression, thus eliminating this potential confounder.

We show here that targeted ablation of IRF3 in adipocytes recapitulates most of the key metabolic phenotypes noted in the global $\mathrm{Irf3}^{-/-}$mice. FI3KO mice have enhanced energy expenditure due to adipose thermogenesis, and are resistant to the deleterious effects of high-fat feeding. Conversely, mice that overexpress an activated allele of IRF3 have reduced adipose thermogenesis, and are less able to defend their body weight in the setting of overnutrition. The effect of IRF3 on insulin action is not dependent on the change in adiposity, since both FI3KO and FI3OE mice have altered insulin sensitivity even when tested before body weights diverge. Interestingly, FI3OE mice do not display insulin resistance in the absence of HFD, suggesting that some other factor associated with high-fat feeding, such as hyperglycemia or hyperinsulinemia, must be present for the insulin resistance to manifest. These studies are the first, to our knowledge, to definitively implicate IRF3 in the function of nonimmune cells.

One interesting feature of our IRF3 gain- and loss-of-function models is that the effect on thermogenic gene expression is seen in iWAT, but not BAT. This finding is in line with other studies that have identified regulators of iWAT browning with minimal effects on BAT function, including MAPK kinase 6 (MKK6), apoptosis signal-regulating kinase 1 (ASK1), laminin subunit $\alpha 4$ (LAMA4), Notch, and PR domain containing 16 (PRDM16) (41, 56, 58-60). In the last example, redundancy with PRDM3 likely explains this phenomenon, as the loss of PRDM16 is not seen in BAT unless PRDM3 is targeted simultaneously (61). By analogy, a related IRF, such as IRF7, might compensate for the loss of IRF3 in BAT, but not in iWAT. We do not favor this hypothesis, however, because (a) gain or loss of IRF3 has the same magnitude of effect on Isg15 expression in BAT and iWAT, and (b) Isg15 ${ }^{-/}$mice exhibit reduced Ucp1 expression in both BAT and iWAT. Rather, our data support the concept that sensitivity to ISG15 differs in the two depots. In this scenario, when the Isg15 gene is ablated, both depots are affected equally, but when the upstream regulator Irf3 is targeted, the small amount of ISG15 that remains is sufficient to control thermogenesis in BAT but not in iWAT. This hypothesis remains speculative but will be the subject of future studies.
IRF3 is a transcription factor, and our unbiased gene expression analysis suggested several target genes, most of which are members of a well-studied group of interferon-stimulated genes, or ISGs. We focused on ISG15 because both it and its associated E3 ligase (Herc6) were among the genes most highly induced by IRF3 in adipocytes. Our subsequent studies showed that loss of ISG15 prevents IRF3 from inhibiting thermogenic gene expression, and that overexpression of ISG15 suppresses thermogenic gene expression, even in the absence of IRF3. Furthermore, mice lacking ISG15 display enhanced thermogenesis and resistance to weight gain on HFD. Taken together, our data demonstrate that ISG15 is a major effector of the antithermogenic action of IRF3. Other transcriptional targets of IRF3 may also play a role; ablation of the IRF3 target gene Rsad2 also leads to enhanced adipose thermogenesis by an unknown mechanism (62). We also note that ISG15 seems to regulate glycemia and insulin action only indirectly, i.e., mediated through changes in adiposity. This is not the case with IRF3; IRF3 must therefore regulate additional genes besides ISG15 that influence insulin action.

ISG15 is a ubiquitin-like protein whose expression and conjugation to targets (ISGylation) are induced by type I interferon (63, $64)$, viral and bacterial infections $(29,65)$, lipopolysaccharide (LPS) (66), and certain genotoxic stressors (67). ISG15 uses a specific set of enzymes to catalyze its covalent attachment and removal from target proteins, including UBE1L as the E1-activating enzyme, UBCH8 as the E2 conjugase, and HERC5 as the E3 ligase (68). Mice do not have a Herc5 gene, and instead use the closely related HERC6 (30). USP18 acts as the major ISG15-specific deconjugating isopeptidase in both humans and mice (69).

Although ISG15 is best known as a ubiquitin-like substrate, it can also act in its free form. This may involve noncovalent binding of ISG15 to enzymes, inhibiting the antiviral response and autophagy (70, 71). ISG15 can also be exported from the cell to act as a cytokine-like signaling molecule, and has been detected in the serum of patients treated with interferon, and in virally infected mice (7274). Secreted ISG15 binds to lymphocyte function-associated antigen 1 (LFA1) on the surface of immune cells, stimulating the release of IL-10 and IFN- $\gamma$, promoting dendritic cell maturation, and acting as a neutrophil chemotactic factor (34). We therefore wanted to clarify whether the antithermogenic actions of ISG15 require conjugation to target proteins, i.e., ISGylation. Our experiments indicate that this is the case. First, ISGylation is increased in adipocytes after high-fat feeding. Second, the repression of Ucp1 observed in cells bearing the activated IRF3-2D allele is reversed by coexpression of USP18. Finally, deletion of HERC6 blocks the actions of the IRF3-2D allele on Ucp1 expression in vivo. Taken together, these data indicate that ISG15 suppresses thermogenic gene expression via covalent conjugation to target proteins, and not in its free form.

Identifying the targets of ISG15 in cells is made difficult by the inability of current antibodies to immunoprecipitate ISGylated proteins. Nonetheless, a few groups have identified an "ISGylome" using either labeled ISG15 (as we do here) or a subtraction-based method in which diglycine adducts (used by both ISG15 and ubiquitin) are identified in $I s g 15^{-/-}$and wild-type cells $(33,75-77)$. We identified 529 ISGylated proteins in adipocytes, most of which are involved in carbohydrate metabolism, stress response, and cell structure. In particular, we noted that almost all glycolytic 
enzymes are ISGylated in adipocytes, consistent with findings that these enzymes are ISGylated in other cell types $(33,77)$.

ISGylation is believed to act by changing the steady-state levels of target viral proteins by proteasomal destruction, in a manner analogous to ubiquitin (78). Some cellular proteins, on the other hand, appear to be stabilized by ISGylation. One example is IRF3, thus enabling a prolonged antiviral response (79). In our experiments, we do not see any change in the steady-state level of glycolytic enzymes in $I s g 15^{-/}$adipocytes despite a large increase in glycolytic flux, suggesting that ISGylation affects enzyme activity and not protein amounts.

Thermogenesis is a highly energetic process, and beige and brown adipocytes consume enormous quantities of substrate when activated. The dominant fuel source for thermogenesis is lipid from white adipose tissue (80); intracellular lipolysis within brown adipocytes plays only a minor role in fueling nonshivering thermogenesis $(81,82)$. Glucose is also a potential fuel for adipose thermogenesis, although early studies suggested that glucose is not a major thermogenic substrate. Experiments in isolated brown adipocytes or using arteriovenous differences in glucose concentration suggested that only $2 \%-16 \%$ of fuel derives from glucose (8385). As one might expect, the importance of glucose as a substrate for thermogenesis increases under artificial conditions where fatty acid oxidation is inhibited $(86,87)$. Cold exposure and $\beta$-adrenergic stimulation also increase glucose uptake in human and rodent BAT $(81,82,88,89)$, and a subset of beige adipocytes with distinct developmental origins has recently been discovered to have a particular dependence on glycolysis (90).

Despite the relatively minor role of glucose as a thermogenic substrate, a consensus has been building that glycolysis is critical for the normal functioning of brown fat. Thus, blocking glycolysis in mouse brown adipocytes severely impairs thermogenesis (35, 36). Furthermore, mice lacking mTORC2 in adipose tissues have reduced thermogenic capacity, which is restored upon overexpression of the glycolytic enzyme Hk2 (91). Similarly, the BAT-enriched NADPH oxidase AIFM2 promotes glycolysis by maintaining a high $\mathrm{NAD}^{+} / \mathrm{NADPH}$ ratio, and is required for normal cold-induced thermogenesis (37). Consistent with this, the expression of some glycolytic enzymes is induced in BAT of cold-exposed mice $(92,93)$.

If glucose is not a critical substrate under normal circumstances, then why is glycolysis required for thermogenesis? The answer is not clear but appears to involve the production of lactate. Cold and catecholamine stimulation increases serum lactate in lambs and mice $(94,95)$. Furthermore, lactate infusion into healthy humans increases energy expenditure (96). Lactate is itself a mitochondrial fuel source, of course, although measurements of the arteriovenous gradient in humans and rodents suggest that BAT is a net producer, rather than a consumer, of lactate $(83,97)$. Nonetheless, this does not preclude lactate from being a quantitatively important fuel source under certain conditions. Thermogenic activity is blocked, for example, when MCT1, the major effector of lactate entry into mitochondria, is inhibited (35). In addition to serving as a potential source of electrons, there are other ways that lactate could drive thermogenesis. Lactate generates reactive oxygen species, which promote the induction of brown-like genes in white adipocytes (95), presumably enabling the cells to reduce redox stress (98). Lactate has also been reported to have pro-adipogenic effects, at least in models of white adipocyte formation (99). Given that many beige adipocytes form by de novo adipogenesis in cold conditions (100), reduced lactate could impair beige thermogenesis.

In our studies, lactate levels are reduced by overexpression of IRF3 or ISG15, and are rescued by USP18. A direct role for lactate is strongly suggested by the fact that an LDHA inhibitor recapitulates the effects of IRF3 and ISG15 in beige adipocytes, and repletion of lactate rescues the effect of IRF3 overexpression. We further identify the specific lysine residue that is bound by ISG15 (K57), and demonstrate a requirement for this residue to mediate the antithermogenic effects of ISG15. Our finding that the $\mathrm{NAD}^{+} / \mathrm{NADH}$ ratio is increased in Isg15-knockout adipocytes is also consistent with a major role for LDHA, as LDHA has preferential affinity for pyruvate over lactate and converts NADH to $\mathrm{NAD}^{+}(101)$.

Our discovery that IRF3 reduces glycolysis is seemingly at odds with reports that TLR ligands, such as LPS, promote a metabolic switch from oxidative phosphorylation to glycolysis in macrophages, resulting in cell activation and polarization to a proinflammatory phenotype (102-104). These studies used macrophage cell lines and/or bone marrow-derived macrophages, however, which do not represent the diversity of macrophage populations in vivo. For example, LPS does not increase glycolysis in tissue-resident macrophages, and glycolysis is not required for the release of proinflammatory cytokines (105). Thus, TLR4 signaling does not activate glycolysis in all cell types. Our data clearly reveal IRF3- and ISG15-dependent suppression of glycolysis in adipocytes, and further work may reveal whether adipocytes are unique in this respect.

Our study addresses a critical issue in the pathophysiology of obesity, as suppression of adipose thermogenesis during overnutrition impedes normal weight homeostasis. Although inflammation has been considered as a leading candidate for an obesity-dependent brake on adipose thermogenesis, the mechanism has been obscure. We provide evidence that IRF3 is a dominant driver of this effect in adipocytes, through induction of ISG15 and its conjugation to glycolytic enzymes. The subsequent suppression of glycolytic flux reduces lactate and inhibits thermogenic gene expression and energy expenditure. This pathway may be amenable to pharmacological intervention, such as by inhibition of HERC5/6 or activation of USP18, with the potential to unleash adipose thermogenesis.

\section{Methods}

Further information can be found in Supplemental Methods.

Study approval. All animal studies were approved by the Institutional Animal Care and Use Committee of Beth Israel Deaconess Medical Center. Human studies were approved by the ethics committee of Dasman Diabetes Institute, Kuwait. All participants gave written informed consent.

All original RNA-Seq data were deposited in the NCBI's Gene Expression Omnibus database (GEO GSE155019).

\section{Author contributions}

SY, MK, RA, and EDR conceived the project. RA procured human tissue. SY, MK, MJ, SK, and RA performed experiments. SY, MJ, $\mathrm{HX}$, and CJ analyzed data. Data curation was performed by SY, MJ, HX, CJ, and EC. SY and EDR wrote the manuscript. Data visualization was provided by SY, MJ, and HX. RA and EDR acquired funding. EDR supervised the project. 


\section{Acknowledgments}

We thank Lilliana Radoshevich for providing reagents (pcDNA3.1hUbe1L, pcDNA3.1-Ubch8, and pTriEx2-hHERC5) and Shingo Kajimura for human brown adipocytes. This work was supported by NIH grants R01 DK085171, R01 DK102173, R01 DK102170, and R01 DK1113669 to EDR; the Claudia Adams Barr Program, NIH grant R01 DK123095, and American Heart Association grant 17SDG33660660 to EC; a National Cancer Center postdoctoral fellowship to HX; and grant RA-AML-2014-016 from the Kuwait Foundation for the Advancement of Sciences (KFAS) to RA. We thank the Boston Nutrition Obesity Research Center Functional Genomics and Bioinformatics Core, the Beth Israel Deaconess Medical Center (BIDMC) Histology Core, and the BIDMC Energy Homeostasis Core. We also thank the Boston Children's Hospital Viral Core for AAV packaging.

Address correspondence to: Evan D. Rosen, Beth Israel Deaconess Medical Center, Harvard Medical School, 330 Brookline Avenue, E/ CLS743, Boston, Massachusetts 02215, USA. Phone: 617.735.3221; Email:erosen@bidmc.harvard.edu.
1. Keesey RE, Powley TL. Body energy homeostasis. Appetite. 2008;51(3):442-445.

2. Rosenbaum M, et al. Effects of experimental weight perturbation on skeletal muscle work efficiency in human subjects. Am J Physiol Regul Integr Comp Physiol.2003;285(1):R183-R192.

3. Rosenbaum M, et al. Long-term persistence of adaptive thermogenesis in subjects who have maintained a reduced body weight. Am JClin Nutr. 2008;88(4):906-912.

4. Leibel RL, et al. Changes in energy expenditure resulting from altered body weight. NEngl JMed. 1995;332(10):621-628.

5. Sims EA, et al. Endocrine and metabolic effects of experimental obesity in man. Recent Prog Horm Res. 1973;29:457-496.

6. van Marken Lichtenbelt WD, et al. Cold-activated brown adipose tissue in healthy men. $N$ Engl JMed. 2009;360(15):1500-1508.

7. Harms M, Seale P. Brown and beige fat: development, function and therapeutic potential. Nat Med. 2013;19(10):1252-1263.

8. Rosen ED, Spiegelman BM. What we talk about when we talk about fat. Cell. 2014;156(1-2):20-44.

9. Goto T, et al. Proinflammatory cytokine interleukin-1 $\beta$ suppresses cold-induced thermogenesis in adipocytes. Cytokine. 2016;77:107-114.

10. Sakamoto T, et al. Inflammation induced by RAW macrophages suppresses UCP1 mRNA induction via ERK activation in 10T1/2 adipocytes. Am J Physiol Cell Physiol. 2013;304(8):C729-C738.

11. Sakamoto T, et al. Macrophage infiltration into obese adipose tissues suppresses the induction of UCP1 level in mice. Am J Physiol Endocrinol Metab. 2016;310(8):E676-E687.

12. Nisoli E, et al. Tumor necrosis factor alpha mediates apoptosis of brown adipocytes and defective brown adipocyte function in obesity. Proc Natl Acad Sci US A. 2000;97(14):8033-8038.

13. Chung KJ, et al. A self-sustained loop of inflammation-driven inhibition of beige adipogenesis in obesity. Nat Immunol. 2017;18(6):654-664.

14 . Bae J, et al. Activation of pattern recognition receptors in brown adipocytes induces inflammation and suppresses uncoupling protein 1 expression and mitochondrial respiration. Am J Physiol Cell Physiol. 2014;306(10):C918-C930.

15. Okla M, et al. Activation of Toll-like receptor 4 (TLR4) attenuates adaptive thermogenesis via endoplasmic reticulum stress. JBiol Chem. 2015;290(44):26476-26490.

16. Gao Z, et al. P65 inactivation in adipocytes and macrophages attenuates adipose inflammatory response in lean but not in obese mice. Am JPhysiol
Endocrinol Metab. 2015;308(6):E496-E505.

17. Tang T, et al. Uncoupling of inflammation and insulin resistance by NF-kappaB in transgenic mice through elevated energy expenditure. JBiol Chem. 2010;285(7):4637-4644.

18. Minegishi $Y$, et al. Deletion of nuclear factor- $\kappa B$ p50 upregulates fatty acid utilization and contributes to an anti-obesity and high-endurance phenotype in mice. Am JPhysiol Endocrinol Metab. 2015;309(6):E523-E533.

19. Honda K, Taniguchi T. IRFs: master regulators of signalling by Toll-like receptors and cytosolic pattern-recognition receptors. Nat Rev Immunol. 2006;6(9):644-658.

20. Eguchi J, et al. Interferon regulatory factors are transcriptional regulators of adipogenesis. Cell Metab. 2008;7(1):86-94.

21. Kong $X$, et al. IRF4 is a key thermogenic transcriptional partner of PGC-1 $\alpha$. Cell. 2014;158(1):69-83.

22. Eguchi J, et al. Transcriptional control of adipose lipid handling by IRF4. Cell Metab. 2011;13(3):249-259.

23. Kumari M, et al. IRF3 promotes adipose inflammation and insulin resistance and represses browning. JClin Invest. 2016;126(8):2839-2854.

24. Stegh AH, et al. Glioma oncoprotein Bcl2L12 inhibits the p53 tumor suppressor. Genes Dev. 2010;24(19):2194-2204.

25. Stegh AH, et al. Bcl2L12 inhibits post-mitochondrial apoptosis signaling in glioblastoma. Genes Dev. 2007;21(1):98-111.

26. Nakajima A, et al. Cell type-dependent proapoptotic role of Bcl2L12 revealed by a mutation concomitant with the disruption of the juxtaposed Irf3 gene. Proc Natl Acad Sci US A. 2009;106(30):12448-12452.

27. Ganeshan K, Chawla A. Warming the mouse to model human diseases. Nat Rev Endocrinol. 2017;13(8):458-465.

28. Yanai $\mathrm{H}$, et al. Revisiting the role of IRF3 in inflammation and immunity by conditional and specifically targeted gene ablation in mice. Proc Natl Acad SciUS A. 2018;115(20):5253-5258.

29. Yuan W, Krug RM. Influenza B virus NS1 protein inhibits conjugation of the interferon (IFN)induced ubiquitin-like ISG15 protein. EMBOJ. 2001;20(3):362-371.

30. Oudshoorn D, et al. HERC6 is the main E3 ligase for global ISG15 conjugation in mouse cells. PLoS One. 2012;7(1):29870.

31. Sarasin-Filipowicz M, et al. Alpha interferon induces long-lasting refractoriness of JAK-STAT signaling in the mouse liver through induction of USP18/ UBP43. Mol Cell Biol. 2009;29(17):4841-4851.

32. Shinoda K, et al. Genetic and functional characterization of clonally derived adult human brown adipocytes. Nat Med.2015;21(4):389-394.

33. Zhao C, et al. Human ISG15 conjugation targets both IFN-induced and constitutively expressed proteins functioning in diverse cellular pathways. Proc Natl Acad Sci U S A . 2005;102(29):10200-10205.

34. Swaim CD, et al. Extracellular ISG15 signals cytokine secretion through the LFA-1 integrin receptor. Mol Cell. 2017;68(3):581-590.

35. Jeong JH, et al. Intracellular glycolysis in brown adipose tissue is essential for optogenetically induced nonshivering thermogenesis in mice. Sci Rep. 2018;8(1):6672.

36. Winther $\mathrm{S}$, et al. Restricting glycolysis impairs brown adipocyte glucose and oxygen consumption. Am J PhysiolEndocrinol Metab. 2018;314(3):E214-E223.

37. Nguyen HP, et al. Aifm2, a NADH oxidase, supports robust glycolysis and is required for cold- and diet-induced thermogenesis. Mol Cell. 2020;77(3):600-617.

38. Semenza GL, et al. Hypoxia response elements in the aldolase A, enolase 1 , and lactate dehydrogenase A gene promoters contain essential binding sites for hypoxia-inducible factor 1. J Biol Chem. 1996;271(51):32529-32537.

39. Platt RJ, et al. CRISPR-Cas9 knockin mice for genome editing and cancer modeling. Cell. 2014;159(2):440-455

40. Cederberg A, et al. FOXC2 is a winged helix gene that counteracts obesity, hypertriglyceridemia, and diet-induced insulin resistance. Cell. 2001;106(5):563-573.

41. Seale P, et al. Prdm16 determines the thermogenic program of subcutaneous white adipose tissue in mice. JClin Invest. 2011;121(1):96-105.

42. Kopecky J, et al. Expression of the mitochondrial uncoupling protein gene from the aP2 gene promoter prevents genetic obesity. JClin Invest. 1995;96(6):2914-2923.

43. Saito M, et al. High incidence of metabolically active brown adipose tissue in healthy adult humans: effects of cold exposure and adiposity. Diabetes. 2009;58(7):1526-1531.

44. Cypess AM, et al. Identification and importance of brown adipose tissue in adult humans. NEngl JMed. 2009;360(15):1509-1517.

45. Claussnitzer M, et al. FTO obesity variant circuitry and adipocyte browning in humans. NEngl JMed. 2015;373(10):895-907.

46. McGregor RA, et al. Time-course microarrays reveal modulation of developmental, lipid metabolism and immune gene networks in intrascapular brown adipose tissue during the development of diet-induced obesity. Int JObes (Lond). 2013;37(12):1524-1531. 
47. Diaz-Delfin J, et al. TNF- $\alpha$ represses $\beta$-Klotho expression and impairs FGF21 action in adipose cells: involvement of JNK1 in the FGF21 pathway. Endocrinology.2012;153(9):4238-4245.

48. Chiang SH, et al. The protein kinase IKKepsilon regulates energy balance in obese mice. Cell. 2009;138(5):961-975.

49. Mowers J, et al. Inflammation produces catecholamine resistance in obesity via activation of PDE3B by the protein kinases IKKE and TBK1. Elife. 2013;2:e01119.

50. Okla M, et al. Inhibitory effects of Toll-like receptor 4, NLRP3 inflammasome, and interleukin-1 $\beta$ on white adipocyte browning. Inflammation . 2018;41(2):626-642.

51. Han MS, et al. Regulation of adipose tissue inflammation by interleukin 6. Proc Natl Acad SciUS A. 2020;117(6):2751-2760.

52. Qing $\mathrm{H}$, et al. Origin and function of stress-induced IL-6 in murine models. Cell. 2020;182(2):372-387.

53. Chen Q, et al. Regulation and function of the cGASSTING pathway of cytosolic DNA sensing. Nat Immunol. 2016;17(10):1142-1149.

54. Honda K, et al. Type I interferon [corrected] gene induction by the interferon regulatory factor family of transcription factors. Immunity. 2006;25(3):349-360.

55 . Reilly SM, et al. An inhibitor of the protein kinases TBK1 and IKK- $\varepsilon$ improves obesity-related metabolic dysfunctions in mice. Nat Med.2013;19(3):313-321.

56. Lucchini FC, et al. ASK1 inhibits browning of white adipose tissue in obesity. Nat Commun . 2020;11(1):1642.

57. Zhao P, et al. TBK1 at the crossroads of inflammation and energy homeostasis in adipose tissue. Cell. 2018;172(4):731-743.

58. Bi P, et al. Inhibition of Notch signaling promotes browning of white adipose tissue and ameliorates obesity. Nat Med. 2014;20(8):911-918.

59. Matesanz N, et al. MKK6 controls T3-mediated browning of white adipose tissue. Nat Commun 2017;8(1):856.

60. Vaicik MK, et al. The absence of laminin $\alpha 4$ in male mice results in enhanced energy expenditure and increased beige subcutaneous adipose tissue. Endocrinology. 2018;159(1):356-367.

61. Harms MJ, et al. Prdm16 is required for the maintenance of brown adipocyte identity and function in adult mice. Cell Metab. 2014;19(4):593-604.

62. Eom J, et al. Intrinsic expression of viperin regulates thermogenesis in adipose tissues. Proc Natl Acad Sci US A. 2019;116(35):17419-17428.

63. Korant $\mathrm{BD}$, et al. Interferon-induced proteins. Purification and characterization of a 15,000-dalton protein from human and bovine cells induced by interferon. J Biol Chem. 1984;259(23):14835-14839.

64. Haas AL, et al. Interferon induces a 15-kilodalton protein exhibiting marked homology to ubiquitin. J Biol Chem. 1987;262(23):11315-11323.

65. Radoshevich L, et al. ISG15 counteracts Listeria monocytogenes infection. Elife. 2015;4:e06848.

66. Malakhova O, et al. Lipopolysaccharide activates the expression of ISG15-specific protease UBP43 via interferon regulatory factor 3 . J Biol Chem. 2002;277(17):14703-14711.

67. Liu M, et al. Camptothecin induces the ubiquitin-like protein, ISG15, and enhances ISG15 conjugation in response to interferon. JInterferon Cytokine Res. 2004;24(11):647-654.
68. Zhang D, Zhang DE. Interferon-stimulated gene 15 and the protein ISGylation system. JInterferon Cytokine Res. 2011;31(1):119-130.

69. Malakhov MP, et al. UBP43 (USP18) specifically removes ISG15 from conjugated proteins. J Biol Chem. 2002;277(12):9976-9981.

70. Okumura A, et al. ISG15 inhibits Ebola VP40 VLP budding in an L-domain-dependent manner by blocking Nedd4 ligase activity. Proc Natl Acad SciU SA. 2008;105(10):3974-3979.

71. Nakashima H, et al. Interferon-stimulated gene 15 (ISG15) and ISG15-linked proteins can associate with members of the selective autophagic process, histone deacetylase 6 (HDAC6) and SQSTM1/p62. J Biol Chem. 2015;290(3):1485-1495.

72. Knight E Jr., Cordova B. IFN-induced 15-kDa protein is released from human lymphocytes and monocytes. JImmunol. 1991;146(7):2280-2284.

73. D'Cunha J, et al. In vitro and in vivo secretion of human ISG15, an IFN-induced immunomodulatory cytokine. JImmunol. 1996;157(9):4100-4108.

74. Werneke SW, et al. ISG15 is critical in the control of Chikungunya virus infection independent of UbE1L mediated conjugation. PLoS Pathog. 2011;7(10):1002322.

75. Lorenz TC, et al. High-throughput protein extraction and immunoblotting analysis in Saccharomyces cerevisiae. Methods Mol Biol. 2008;457:13-27.

76. Giannakopoulos NV, et al. Proteomic identification of proteins conjugated to ISG15 in mouse and human cells. Biochem Biophys Res Commun. 2005;336(2):496-506.

77. Zhang Y, et al. The in vivo ISGylome links ISG15 to metabolic pathways and autophagy upon Listeria monocytogenes infection. Nat Commun. 2019;10(1):5383.

78. Durfee LA, et al. The ISG15 conjugation system broadly targets newly synthesized proteins: implications for the antiviral function of ISG15. Mol Cell. 2010;38(5):722-732.

79. Shi HX, et al. Positive regulation of interferon regulatory factor 3 activation by Herc 5 via ISG15 modification. Mol Cell Biol. 2010;30(10):2424-2436.

80. Honnor RC, et al. cAMP-dependent protein kinase and lipolysis in rat adipocytes. II. Definition of steady-state relationship with lipolytic and antilipolytic modulators. J Biol Chem. 1985;260(28):15130-15138.

81. Shin H, et al. Lipolysis in brown adipocytes is not essential for cold-induced thermogenesis in mice. Cell Metab. 2017;26(5):764-777.

82. Schreiber R, et al. Cold-induced thermogenesis depends on ATGL-mediated lipolysis in cardiac muscle, but not brown adipose tissue. Cell Metab. 2017;26(5):753-763.

83. Ma SW, Foster DO. Uptake of glucose and release of fatty acids and glycerol by rat brown adipose tissue in vivo. Can JPhysiol Pharmacol.1986;64(5):609-614.

84. Isler D, et al. Glucose metabolism in isolated brown adipocytes under beta-adrenergic stimulation. Quantitative contribution of glucose to total thermogenesis. Biochem J. 1987;245(3):789-793.

85. Saggerson ED, et al. Lipogenesis in rat brown adipocytes. Effects of insulin and noradrenaline, contributions from glucose and lactate as precursors and comparisons with white adipocytes. Biochem J 1988;251(3):701-709.

86. Held NM, et al. Pyruvate dehydrogenase complex plays a central role in brown adipocyte energy expenditure and fuel utilization during short-term beta-adrenergic activation. Sci Rep. 2018;8(1):9562.

87. Caron A, et al. Loss of UCP2 impairs cold-induced non-shivering thermogenesis by promoting a shift toward glucose utilization in brown adipose tissue. Biochimie. 2017;134:118-126.

88. Orava J, et al. Different metabolic responses of human brown adipose tissue to activation by cold and insulin. Cell Metab. 2011;14(2):272-279.

89. Ouellet V, et al. Brown adipose tissue oxidative metabolism contributes to energy expenditure during acute cold exposure in humans. JClin Invest. 2012;122(2):545-552.

90. Chen Y, et al. Thermal stress induces glycolytic beige fat formation via a myogenic state. Nature. 2019;565(7738):180-185.

91. Albert V, et al. mTORC2 sustains thermogenesis via Akt-induced glucose uptake and glycolysis in brown adipose tissue. EMBO Mol Med. 2016;8(3):232-246

92. Basse AL, et al. Regulation of glycolysis in brown adipocytes by HIF-1 $\alpha$. Sci Rep. 2017;7(1):4052.

93. Hao Q, et al. Transcriptome profiling of brown adipose tissue during cold exposure reveals extensive regulation of glucose metabolism. Am J Physiol Endocrinol Metab. 2015;308(5):E380-E392.

94. Alexander $\mathrm{G}$, et al. Changes in plasma glucose, lactate and free fatty acids in lambs during summit metabolism and treatment with catecholamines. JPhysiol. 1968;198(2):277-289.

95. Carriere A, et al. Browning of white adipose cells by intermediate metabolites: an adaptive mechanism to alleviate redox pressure. Diabetes. 2014;63(10):3253-3265.

96. Ferrannini E, et al. Metabolic and thermogenic effects of lactate infusion in humans. Am JPhysiol. 1993;265(3 pt 1):E504-E512.

97. Weir $\mathrm{G}$, et al. Substantial metabolic activity of human brown adipose tissue during warm conditions and cold-induced lipolysis of local triglycerides. Cell Metab. 2018;27(6):1348-1355.

98. Jeanson $\mathrm{Y}$, et al. A new role for browning as a redox and stress adaptive mechanism? Front Endocrinol (Lausanne). 2015;6:158.

99. Harada N, et al. Stereoselective effects of lactate enantiomers on the enhancement of 3T3-L1 adipocyte differentiation. Biochem Biophys Res Commun. 2018;498(1):105-110.

100. Shao M, et al. Cellular origins of beige fat cells revisited. Diabetes. 2019;68(10):1874-1885.

101. Valvona CJ, et al. The regulation and function of lactate dehydrogenase A: therapeutic potential in brain tumor. Brain Pathol. 2016;26(1):3-17.

102. Kelly B, O'Neill LA. Metabolic reprogramming in macrophages and dendritic cells in innate immunity. Cell Res. 2015;25(7):771-784.

103. Krawczyk CM, et al. Toll-like receptor-induced changes in glycolytic metabolism regulate dendritic cell activation. Blood. 2010;115(23):4742-4749.

104. Pantel A, et al. Direct type I IFN but not MDA5/ TLR3 activation of dendritic cells is required for maturation and metabolic shift to glycolysis after poly IC stimulation. PLoS Biol. 2014;12(1):1001759.

105. Woods PS, et al. Tissue-resident alveolar macrophages do not rely on glycolysis for LPS-induced inflammation. Am J Respir Cell Mol Biol. 2020;62(2):243-255. 